\title{
Competitive Stimulus Interactions within Single Response Fields of Superior Colliculus Neurons
}

\author{
Xiaobing $\mathrm{Li}^{1}$ and Michele A. Basso ${ }^{1,2}$ \\ Departments of ${ }^{1}$ Physiology and ${ }^{2}$ Ophthalmology and Visual Sciences, University of Wisconsin-Madison Medical School, Madison, Wisconsin 53706
}

In addition to its role in saccade generation, the superior colliculus (SC) is involved in target selection, saccade selection, and shifting the focus of spatial attention. Here, we investigated the influence of saccade selection on sensory interactions within single response fields (RFs) of SC neurons. One or two differently shaped stimuli were presented within single RFs of SC neurons, and the shape of a centrally located cue indicated whether and where to make a saccade (Go-Go) or whether to make or withhold a saccade (Go/No-Go). We found that, when two stimuli appeared at different locations within a single RF, SC neuronal activity was reduced compared with when a single stimulus appeared in isolation within the center of the RF in both the Go-Go and Go/No-Go tasks. In both tasks, a subsequent cue indicating one stimulus as a saccade target reduced the influence of the second stimulus located within the RF. We found that the time course of the suppression resulting from the two stimuli was $\sim 130 \mathrm{~ms}$, a time close to that seen in cortex. Finally, we found that the influence of two stimuli within single RFs of SC neurons changed over time in both the Go-Go and the Go/No-Go tasks. Initially, the neurons averaged the influence of two stimuli. As the trial progressed, the $\mathrm{SC}$ neurons signaled only the saccade vector that was produced. We conclude that cues to shift gaze, like attention, modulate the influence of sensory interactions, providing additional support for the linkage between attention and saccade selection.

Key words: saccade; spatial attention; target selection; population coding; decision; electrophysiology

\section{Introduction}

The superior colliculus (SC) plays a critical role in eye movement generation and the preceding events such as visual target selection for saccades (Basso and Wurtz, 1998; Port and Wurtz, 2003; McPeek and Keller, 2004), for smooth pursuit (Krauzlis and Dill, 2002; Carello and Krauzlis, 2004), saccade selection (Glimcher and Sparks, 1992), saccade preparation (Dorris et al., 1997), shifting the focus of spatial attention (Goldberg and Wurtz, 1971, 1972b; Cavanaugh and Wurtz, 2004; Ignashchenkova et al., 2004; Muller et al., 2005), and perceptual decision making (Horwitz and Newsome, 1999, 2001). Psychophysical experiments demonstrate a strong coupling between saccades and shifts of spatial attention (Kowler, 1990; Kowler and Blaser, 1995; Kowler et al., 1995; Deubel and Schneider, 1996; Schneider and Deubel, 2002), suggesting that physiological mechanisms underlying selection for attention and selection for action are shared. How this is implemented within the SC is unknown and is the focus of the current report.

A conceptual model of attention developed from studies in

Received June 24, 2005; revised 0ct. 15, 2005; accepted 0ct. 27, 2005

This work was supported by National Institutes of Health Grant EY013692 and The Esther A. and Joseph Klingenstein Fund. We are grateful to Dr. John H. Reynolds and our colleagues in the laboratory for providing critical comments on a previous version of this manuscript. We thank Drs. Michael Shadlen for sharing Matlab code and John W. McClurkin for analysis software support. We also thank Joshua Smith for expert technical assistance and the anonymous referees for their critical review.

Correspondence should be addressed to Dr. Michele A. Basso, Department of Physiology, University of Wisconsin, Madison Medical School, 1300 University Avenue, Room 127 SM1, Madison, WI 53706. E-mail: michele@physiology.wisc.edu.

DOI:10.1523/JNEUROSCI.3825-05.2005

Copyright $\odot 2005$ Society for Neuroscience 0270-6474/05/2511357-17\$15.00/0 visual areas V4 and inferotemporal cortex IT is called the biased competition model (Desimone and Duncan, 1995; Reynolds et al., 1999). This model has two major predictions. First, when two stimuli appear in a single response field (RF), they will compete. If a single stimulus produces a strong response in a neuron, the addition of a second, weaker stimulus will reduce the response. Second, when attention is directed to one of the two stimuli, the neuronal activity will be dominated by the response of the neuron to that stimulus when it is presented in isolation. Thus, attention appears to modulate sensory responses in cortex (Luck et al., 1997; Reynolds et al., 1999, 2000). Recent experiments in SC and frontal eye field (FEF) using multiple stimuli with large spatial separations demonstrate that neuronal responses are reduced compared with when a single stimulus appears in isolation (Schall, 1991, 1995; Schall et al., 1995; Basso and Wurtz, 1998; McPeek and Keller, 2002), similar to observations made in extrastriate cortex (Motter, 1993, 1994b; Treue and Maunsell, 1996; Luck et al., 1997; Recanzone et al., 1997). In SC, when a peripheral cue indicates which stimulus will be a saccade target, neuronal activity increases to levels measured in single stimulus trials (Basso and Wurtz, 1998), suggesting that a cue to shift gaze can influence sensory interactions arising from the surrounding stimuli.

Here, we test predictions of the biased competition model (Desimone and Duncan, 1995; Reynolds et al., 1999) in individual SC neurons. For SC, the relevant stimulus dimension is location, so we adapted feature attention tasks (Reynolds et al., 1999) to visually guided saccade tasks to determine whether the biased competition model could be generalized to spatial location within the RF of SC neurons. The results demonstrate stimulus 
interactions influencing individual SC neurons. The interactions were modified by cues to shift gaze. Our observations are consistent with the hypothesis that cues to shift gaze, like attention, modulate the influence of sensory interactions, providing additional support for the linkage between attention and saccade selection.

\section{Materials and Methods}

\section{Physiological procedures}

For electrophysiological recording of single neurons and monitoring eye movements, cylinders and eye coils were implanted in four rhesus monkeys (Macaca mulatta) using standard procedures as described previously (Basso and Wurtz, 2002). Anesthesia was induced initially with an intramuscular injection of ketamine $(5.0-15.0 \mathrm{mg} / \mathrm{kg})$. Atropine $(0.5 \mathrm{mg} / \mathrm{kg})$ was provided to minimize salivation. Monkeys were intubated and maintained at a general anesthetic level with isofluorane. A subconjunctival eye coil was implanted (Judge et al., 1980). A plastic head holder for restraint and a cylinder for subsequent microelectrode recording were mounted on the top of the exposed skull and secured with titanium screws and dental acrylic. Plastic hardware allowed subsequent magnetic resonance images to be obtained with minimal artifact. For access to the SC, the recording cylinder was placed stereotaxically on the midline and angled $38^{\circ}$ back so that the electrode penetrations were directed caudorostrally, toward the SC. An antibiotic (Cefadroxil, $25 \mathrm{mg} / \mathrm{kg}$ ) was given $1 \mathrm{~d}$ before the operation and every day for a minimum of $4 \mathrm{~d}$ after the operation. Analgesia was provided by the administration of buprenorphine (0.01-0.03 $\mathrm{mg} / \mathrm{kg}$ ) and Flunixin (1-2 mg/kg) for $48 \mathrm{~h}$ postsurgically as needed. Monkeys recovered for 1-2 weeks before behavioral and physiological recording commenced. All experimental protocols were approved by the University of Wisconsin-Madison Institutional Animal Care and Use Committee and complied with or exceeded standards set by the Public Health Service policy on the humane care and use of laboratory animals.

\section{General behavioral procedures}

We used a real-time experimental data acquisition and visual stimulus generation system (Tempo and VideoSync; Reflective Computing, St. Louis, MO) to create the behavioral paradigms and acquire eye position and single neuron data. Monkeys were trained to sit in a customdesigned primate chair with head fixed during the experimental session (typically $3-5 \mathrm{~h}$ ). Visual stimuli were rear projected on a tangent screen at $51 \mathrm{~cm}$ distance using a digital light projector (DLP) (LP335; Infocus, Wilsonville, OR) with a native resolution of $1024 \times 768$ and operating at $60 \mathrm{~Hz}$. The background luminance was $0.28 \mathrm{~cd} / \mathrm{m}^{2}$. The visual stimulus presentation was controlled by VideoSync software (Reflective Computing) running on a dedicated personal computer $(\mathrm{PC})$ with a $1024 \times 768$ VGA video controller (Computer Boards). The PC was a slave device to the PC used for experimental control and data acquisition. Because there is an inherent time limitation in DLPs (both the vertical refresh rate and the vertical synch pulse), a photocell was placed on the tangent screen, which sent a signal [a transistor-transistor logic (TTL) pulse] to the experimental PC providing an accurate measure of stimulus onset.

\section{Behavioral tasks}

Control task. Because we used different shapes (triangle and square) in our tasks, we initially measured neuronal activity during performance of this task to ensure that SC neurons were not shape selective. The same number of white pixels made up each isoluminant stimulus $(2.17 \mathrm{~cd} /$ $\mathrm{m}^{2}$ ). The size of the stimuli was scaled with the distance from the fovea, either $0.6^{\circ}$ for distances $<15^{\circ}$ or $0.9^{\circ}$ for distances $>15^{\circ}$. Initially, monkeys looked at a centrally located fixation spot for 800-1500 ms. Then, either a triangle or a square was displayed on the screen. The target was placed in the optimal location of the RF of the neuron under study (see below for RF mapping). At the end of this second delay (800-1000 ms), the fixation point was removed, and this cued the monkeys to make a saccade to the target. Monkeys were required to maintain eye position at the target location for $400-600 \mathrm{~ms}$. If they maintained accuracy within a $2^{\circ}$ square electronic window (if the target was farther than $10^{\circ}$, the window was increased to $3-4^{\circ}$ square), they received a liquid reward. Square and triangle trials were randomized. In this task, before training on the other behavioral tasks commenced, we recorded seven buildup and eight visual-tonic neurons. A visual $(0-200 \mathrm{~ms}$ from target onset), a delay (300-800 ms after target onset), and a saccade ( $100 \mathrm{~ms}$ before to $100 \mathrm{~ms}$ after the onset of a saccade) interval were measured to compare the neuronal activity in the two conditions. No SC neuron showed any significant differences in these two conditions in any of the intervals measured ( $t$ test, $p>0.05$; data not shown). Thus, not surprisingly, we concluded that our stimuli were neutral to SC neurons before any training in the tasks.

Go-Go task. After the onset of a centrally located fixation spot for a random time of 1000-1500 ms, either one or two shapes were presented within a single RF of an SC neuron (see below, Response field mapping). The fixation point remained illuminated along with the peripheral stimuli for a random delay of $800-1000 \mathrm{~ms}$, and then the fixation point changed shape to either a triangle or a square (Fig. 1a). After another $800-1000$ ms delay, the fixation point turned back to its original spot, and this was the cue for the monkey to make a saccade to the cued stimulus. If the monkey acquired the target and its eye position remained at that location for $400-600 \mathrm{~ms}$, a liquid reward was provided. The accuracy criterion was $2^{\circ}$ square around the target (if the target was farther than $10^{\circ}$, the criterion was increased to $3-4^{\circ}$ square around the target). The smallest distance between two stimuli was $\sim 2^{\circ}$, whereas the largest was $\sim 25^{\circ}$. Importantly the separation between the two stimuli 
exceeded the size of the windows to discourage averaging saccades and encourage precise saccades.

Each experimental session consisted of four trial types presented in two conditions. The trial types were presented in blocks in one condition ("blocked") and randomly in a second condition ("interleaved"). One trial type was a triangle presented in the RF with a triangle cue presented at the fovea (1T). A second type was a square presented in the RF with a square cue presented at the fovea (1S). A third type presented both the triangle and square stimuli in the RF with a triangle cue at the fovea (2T). In the fourth type, both the triangle and square were presented in the RF, and a square cue was at the fovea (2S). We collected, on average, 20-50 trials for each type.

Go/No-Go task. This task was identical to the Go-Go task, except here, the triangle served as a cue for the Go condition, whereas a square indicated the No-Go condition (Fig. 1b). The square cue indicated that the monkey had to remain fixating at the central spot for 400-600 ms to obtain a fluid reward. In the Go trials, saccades had to be accurate, and we ensured this by making the acceptance criterion defined by the electronic windows nonoverlapping. Thus, averaging saccades were discouraged. We collected, on average, 20-50 trials for each trial type in this condition.

\section{Response field mapping}

A stimulus was moved around to assess qualitatively the boundary and the center of the visual RF of SC neurons. We then placed the triangle stimulus in the approximate center of the RF and a second stimulus (square) at any location around the center but, importantly, within the boundaries of the RF. The placement of the second stimulus was determined empirically by listening and watching the neuronal discharge online. Because we were interested in accessing stimulus interactions, we placed the second stimulus within a region of the RF that was likely to produce suppression, if it existed. However, during data analysis, we discovered that, in some cases, the two stimuli resulted in enhanced responses in the averaged discharge (these neurons can be identified on the population plots in Results). The separation between the two stimuli was scaled by the diameter of the visual RF. For example, if the diameter of the field was $\sim 5^{\circ}$, the two stimuli would be located $\sim 2^{\circ}$ apart; if the diameter was $\sim 10^{\circ}$, then the two stimuli would be located $\sim 5^{\circ}$ apart. We also placed the two stimuli approximately equidistant from the fixation point to keep the amplitude of the vector constant. We did not use neurons with RFs that included the fovea. For some neurons with large $\mathrm{RFs}$, we were unable to determine exactly the distal boundaries of the RF.

\section{Neuronal classification}

We classified neurons as either buildup or visual-tonic in the single target, visually guided saccade task using the following statistical criteria. We computed a baseline measure of activity ( $200 \mathrm{~ms}$ before the onset of the visual target), a delay period activity ( $400 \mathrm{~ms}$ before a cue), and a saccade period activity (100 ms before saccade onset). Using only saccade correct trials, we defined buildup neurons as those neurons with a significantly greater activity in the delay period compared with the baseline $(t$ test, $p<0.05)$ and a significantly greater activity in the saccade period compared with the delay period ( $t$ test, $p<0.05$ ). If a neuron had a visual response and had a significantly greater level of activity in the delay period than the baseline ( $t$ test, $p<0.05$ ) but had no significant difference between the saccade period and the delay period, we classified the neuron as a visual-tonic neuron. Examples of each type are shown in Figure 2.

\section{Data acquisition and analysis}

Using the magnetic search coil technique (Fuchs and Robinson, 1966) (Riverbend Instruments, Birmingham, AL), voltage signals proportional to horizontal and vertical components of eye position were filtered (eight-pole Bessel, $-3 \mathrm{~dB}, 180 \mathrm{~Hz}$ ), digitized at 16-bit resolution, and sampled at $1 \mathrm{kHz}$ (CIO-DAS1602/16; Measurement Computing, Middleboro, MA). The data were saved for off-line analysis using an interactive computer program designed to display and measure eye position and calculate eye velocity. We used an automated procedure to define saccadic eye movements by applying velocity and acceleration criteria of $50 \%$ and $5000 \% \mathrm{~s}^{2}$, respectively. The adequacy of the algorithm was verified on a trial-by-trial basis by the experimenter. Single neurons

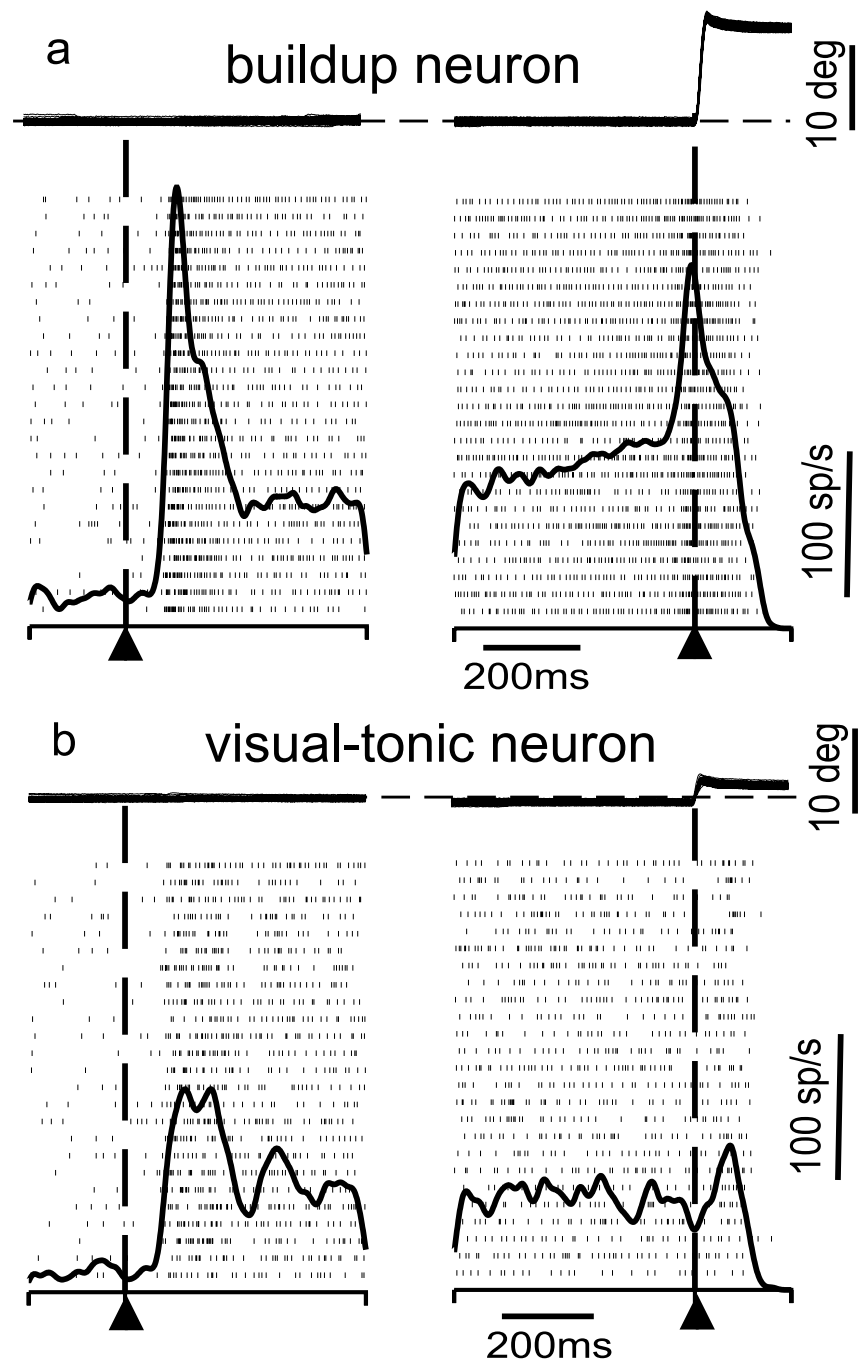

Figure 2. Examples of SC neurons. $\boldsymbol{a}$, An SC buildup neuron during a visually guided saccade. Along the top, eye position traces are plotted against time. The dashed, horizontal line indicates primary position. An upward deflection in the position traces is right, and a downward deflection is left. Traces on the left are aligned on target onset, and traces on the right are aligned on saccade onset. The alignment is indicated by the dashed, vertical line and arrowhead. Below the eye position traces are the raster plots and the superimposed spike density functions ( $\sigma=15$ $\mathrm{ms}$ ). Each tick in the raster represents an action potential, and each row of ticks represents an individual trial in which the monkey made a delayed, visually guided saccade. $\boldsymbol{b}$, Arrangement is the same as in $\boldsymbol{a}$, for an SC visual-tonic neuron.

were recorded with tungsten microelectrodes (Frederick Haer Company, Bowdoinham, ME) with impedances between 0.3 and $1.0 \mathrm{M} \Omega$ measured at $1 \mathrm{kHz}$. Electrodes were aimed at the SC through stainless steel guide tubes held in place by a plastic grid secured to the cylinder (Crist et al., 1988). Action potential waveforms were identified with a window discriminator (Bak Electronics, Germantown, MD) that returned a TTL pulse for each waveform that met voltage and time criteria. The TTL pulses were sent to a digital counter (PC-TIO-10; National Instruments, Austin, TX) and were stored with a $1 \mathrm{~ms}$ resolution.

Statistical analyses and curve fits were performed using Matlab (MathWorks, Natick, MA). Standard parametric descriptive and inferential (ANOVA, $t$ tests with modified Bonferroni corrections) statistics were used (Keppel, 1991). If the data failed to pass normality tests, nonparametric statistics were used. Wilcoxon's signed-rank test was used for sample data analyses. To compare the time course of neuronal activity changes in the different stimulus conditions, we computed receiver operating characteristic (ROC) curves based on signal detection theory (Green and Swets, 1966), similar to that performed by others (Cohn et al., 
1975; Bradley et al., 1987; Britten et al., 1992; Thompson et al., 1996). For each trial, we divided individual spike trains recorded in the two conditions into $5 \mathrm{~ms}$ bins and averaged the discharge rate within the bin. We then computed the probability that the discharge rate exceeded a criterion. The criterion was incremented from 0 to the maximum discharge rate for each $5 \mathrm{~ms}$ bin, and a probability value was computed for each criterion. Therefore, a single point on the ROC curve is produced for each increment in the criterion, and the entire ROC curve is generated from all of the criteria. The area under the ROC curve is a measure of the separation between the two distributions, and the area value provides a measure of the probability that the two curves could be distinguished. However, whether this value is statistically significant is ambiguous. Therefore, we performed a permutation test (Efron and Tibshirani, 1998) to determine whether the areas we measured were statistically reliable. For each $5 \mathrm{~ms}$ bin and each neuron, we randomly sampled the discharge rate 1000 times and generated an ROC curve for each permutation. This resulted in a distribution of ROC areas for every $5 \mathrm{~ms}$ time point. The original ROC area for individual neurons was compared with this distribution of areas to determine whether it fell within or outside of the 95th percentile. If the original value fell outside of the 95th percentile, we determined that the difference between the two curves at that time point was statistically reliable. We then computed a mean separation time from all of the significant times across the sample of neurons.

\section{Results}

We recorded from 151 SC neurons in four monkeys and classified (see Materials and Methods) each as buildup (106) or visualtonic (45). Although variable, we tended to find visual-tonic neurons dorsally in the SC (mean $\pm \mathrm{SD}, 1.1 \pm 0.7 \mathrm{~mm}$ from the surface) and buildup neurons below visual-tonic neurons (mean $\pm \mathrm{SD}, 1.6 \pm 0.6 \mathrm{~mm}$ from the surface). Subsets of these neurons were recorded in different tasks (control, Go-Go and Go/No-Go). In two monkeys, we recorded neurons only during performance of the Go/No-Go task, and, in two monkeys, we recorded during both tasks. Of the two that learned both tasks, one learned the Go/No-Go task first and then learned the Go-Go task, and the second learned the Go-Go task first and then learned the Go/No-Go task. Table 1 shows the breakdown of how many neurons were recorded in each condition and in each task. It also indicates the number of neurons recorded in both tasks. Note that the monkeys did not always complete all of the trial types in all tasks, so particularly in the interleaved conditions, there are often fewer neurons. Also note that the neurons recorded in the control task $(n=15)$ are excluded from the table.

\section{Go-Go task: behavior of buildup neurons}

In most models of competition, neurons with similar properties are mutually excitatory, whereas neurons with dissimilar properties are mutually inhibitory (Desimone and Duncan, 1995). In our task, the presentation of two stimuli within a single SC RF led to the activation of two overlapping populations of neurons (McIlwain, 1975, 1986) reflecting close (generally within $5^{\circ}$ ) but different locations. If stimulus competition exists, we predicted a reduced level of activity in SC neurons in the two stimulus conditions compared with when a single stimulus was presented. We performed this task in blocked and interleaved trial conditions.

To display the results, we constructed mean spike density functions for all of the neurons except those showing statistically significant effects that were opposite our predictions in at least one interval (for description of intervals, see below or Materials and Methods). To assess the variability, the results for the individual neurons across the entire sample are shown in plots, generally in the figures after the spike density functions. We recorded 24 buildup neurons in interleaved conditions of the Go-Go task, and two had opposite effects. Therefore, 22 neurons were used to
Table 1. The breakdown of neurons recorded in each condition and in each task

\begin{tabular}{llllll}
\hline & \multicolumn{2}{l}{ Blocked } & & \multicolumn{2}{l}{ Interleaved } \\
\cline { 2 - 3 } \cline { 5 - 6 } & Go-Go & Go/No-Go & & Go-Go & Go/No-Go \\
\hline $\begin{array}{l}\text { Buildup } \\
n\end{array}$ & 39 & 47 & 24 & 44 \\
$\quad$ Overlapping & 24 & 31 & & 13 \\
$\begin{array}{l}\text { Visual-tonic } \\
n\end{array}$ & 17 & 18 & 9 & \\
$\quad$ Overlapping & 9 & 11 & & \\
\hline
\end{tabular}

Numbers of neurons recorded in the different task conditions are indicated.

$n$ indicates the number of neurons recorded in the four monkeys. Blocked and interleaved indicate the conditions, and $\mathrm{G}_{0} / \mathrm{No} \mathrm{o}_{\mathrm{G}} \mathrm{G}$ and $\mathrm{G}_{0}-\mathrm{G}_{0}$ indicate the task types. Overlapping indicates the number of neurons recorded in both conditions.

construct the mean spike density function shown in Figure $3 a$. Across this sample of buildup neurons, when a single stimulus was presented in the center of the RF, we observed a robust discharge of action potentials (Fig. 3a, left panel, red traces 1T). During the delay period, action potentials were maintained at a lower level (Fig. $3 a$, middle panel, red traces 1T). When the fixation point changed shape, indicating the target for an upcoming saccade, the activity of buildup neurons increased further (Fig. $3 a$, middle panel, red traces $1 \mathrm{~T}$ ). Finally, when the fixation point changed back to its original shape, providing a cue to initiate a saccade to the target, we observed a robust discharge of action potentials (Fig. $3 a$, right panel, red traces $1 \mathrm{~T}$ ). Because the stimulus located at the edge of the RF was also excitatory for the neurons, a qualitatively similar profile of activity was observed (Fig. $3 a$, blue traces 1S), although at a lower level. When these two stimuli were presented simultaneously within the RF, the activity of buildup neurons was reduced (Fig. $3 a$, left panel, green traces $2 \mathrm{~T}$, yellow traces $2 \mathrm{~S}$ ). When the saccade target was identified, the neuronal activity seen in buildup neurons showed two possible profiles. If the stimulus located in the center of the field was indicated, the activity increased (Fig. $3 a$, middle panel, green traces $2 \mathrm{~T}$ ). If the edge stimulus was indicated as the target of the saccade, the activity increased slightly or remained essentially unchanged (Fig. $3 a$, middle panel, yellow traces 2 S). Note, however, that the pair activity was still higher when the cue indicated the center stimulus compared with when the cue indicated the edge stimulus. By the time of saccade initiation, the neuronal activity measured in these two conditions was clearly separate (Fig. $3 a$, right panel, green traces $2 \mathrm{~T}$, yellow traces $2 \mathrm{~S}$ ). When the saccade was made to the center of the field, the activity was statistically indistinguishable for the one or two stimulus conditions across the sample of neurons (Fig. $3 a$, right panel, red traces 1T, green traces $2 \mathrm{~T}$ ). A similar pattern was also seen when the saccade was made to the edge stimulus, although the saccade-related discharge was less robust (Fig. $3 a$, blue traces $1 S$, yellow traces $2 S$ ).

Compared with the interleaved target trial conditions, the neuronal modulations were more robust when the trial conditions were blocked. The results obtained in the blocked target trial condition for a single buildup neuron are shown in Figure $3 b$. We recorded from 39 neurons in the blocked condition ( 24 of these were also recorded in the interleaved condition) (Table 1). Seven neurons showed opposite effects; therefore, the mean spike density function for 32 buildup neurons is shown in Figure $3 c$. In the single neuron as well as the sample data, the separation between the $2 \mathrm{~T}$ and $2 \mathrm{~S}$ conditions was seen even in the initial visual response (Fig. 3b,c, green and yellow traces). This was evident because the monkeys knew in advance which saccade would be required (Basso and Wurtz, 1997). Because of the clarity of the 


\section{buildup neurons - interleaved - Go - Go task}

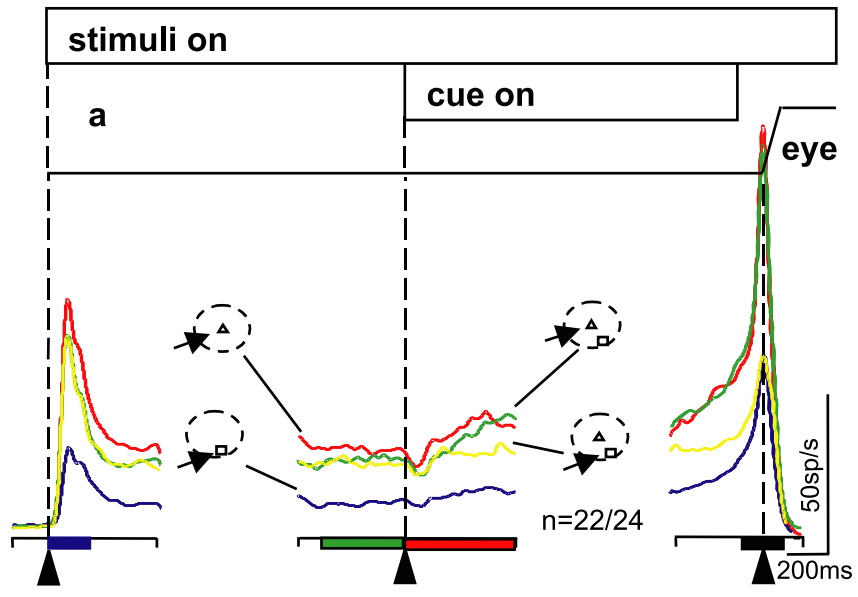

buildup neuron - blocked - Go - Go task

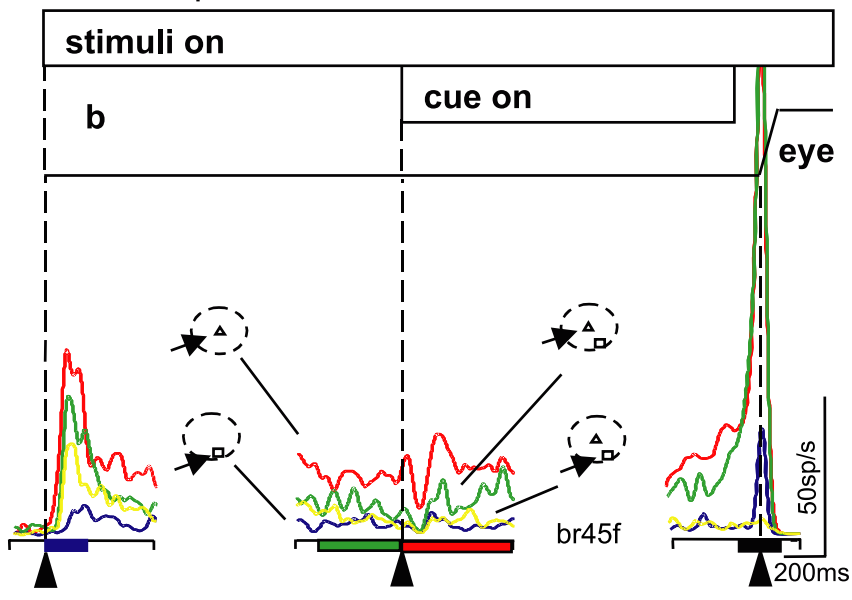

buildup neurons - blocked - Go - Go task

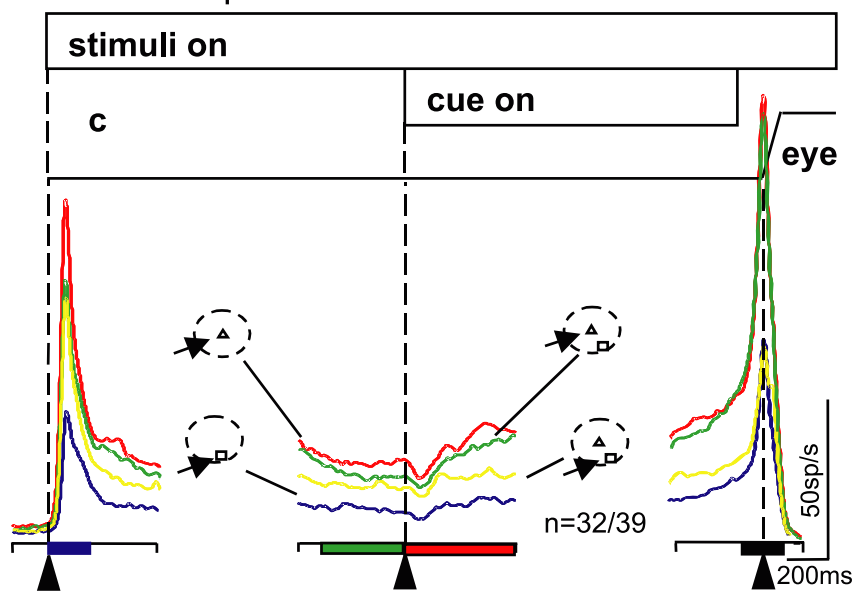

Figure 3. Buildup neurons have reduced activity when two stimuli are in the RF and a central cue biases neuronal activity. $\boldsymbol{a}$, Spike density functions ( $\sigma=15 \mathrm{~ms}$ ) averaged over 22 buildup neurons are shown for each of the four trial types in the interleaved condition. Data are from correctly performed trials. Red traces are from $1 \mathrm{~T}$ trials, blue traces are from $1 \mathrm{~S}$ trials, green traces are from $2 \mathrm{~T}$ trials, and yellow traces are from $2 \mathrm{~S}$ trials. The labeled bars at the top of the figure indicate the temporal arrangement of the task, and the eye position trace labeled "eye" is a schematic. The insets are schematics of the stimulus configurations, and the arrows indicate trial conditions in which saccades were required. The left panel is aligned on the onset of the visual stimuli indicated by the dashed vertical line and arrowhead. The middle panel is aligned on the onset of the cue indicated by the dashed vertical line and arrowhead, and the right panel is aligned on the onset of the saccade, also indicated by a dashed vertical line and arrowhead. effects, we will use examples from the blocked conditions to illustrate most of the rest of the results.

Buildup neuron activity across the sample: two stimulus interactions

Two excitatory stimuli located within a single RF of SC buildup neurons resulted in a suppression of neuronal activity compared with when a single, excitatory stimulus was presented in isolation. To quantify the activity across all of the neurons in our sample during the Go-Go task, we divided the task into four intervals and measured the mean neuronal discharge rate in these intervals. The first $200 \mathrm{~ms}$ interval began at the time the stimuli appeared (visual interval). The second interval (delay 1) was defined as the $400 \mathrm{~ms}$ of activity before the onset of the cue. The third interval began at the time the cue indicated the target and continued for $600 \mathrm{~ms}$ (delay 2). The saccade interval was defined as $100 \mathrm{~ms}$ before to $100 \mathrm{~ms}$ after saccade onset (for saccade detecting algorithm, see Materials and Methods).

In the blocked condition, 29 of 39 (74\%) buildup neurons showed significantly lower responses in the $2 \mathrm{~T}$ condition compared with the $1 \mathrm{~T}$ condition during the visual interval (Fig. $4 a$, filled blue circles, points below the unity line), whereas 13 of 24 $(54 \%)$ showed this in the interleaved condition (Fig. $4 a$, filled blue triangles) ( $t$ test, Bonferroni corrected, $p<0.05$ ). Seventeen of $39(44 \%)$ buildup neurons in blocked trials showed significantly reduced activity for the $2 \mathrm{~T}$ condition compared with the $1 \mathrm{~T}$ condition during delay 1 (Fig. $4 b$, filled green circles), whereas 4 of 24 (17\%) showed this pattern in interleaved trials (Fig. $4 b$, filled green triangles) ( $t$ test, Bonferroni corrected, $p<0.05$ ). During delay 2, 17 of 39 (44\%) showed significantly reduced activity in blocked trials (Fig. $4 c$, filled red circles), and 6 of 24 (25\%) buildup neurons had significantly reduced activity (Fig. $4 b$, filled red triangles) in interleaved trials ( $t$ test, Bonferroni corrected, $p<0.05$ ). Despite the fact that the same saccade was made in the $2 \mathrm{~T}$ and $1 \mathrm{~T}$ conditions, 8 of $39(21 \%)$ neurons had significantly lower activity during the saccade interval for the $2 \mathrm{~T}$ condition in blocked trials (Fig. $4 d$, filled black circle), and 4 of 24 $(17 \%)$ showed this in interleaved trials (Fig. $4 d$, filled black triangle) ( $t$ test, Bonferroni corrected, $p<0.05)$. Across all neurons in the sample, the differences were significantly different for all intervals except the saccade interval (Wilcoxon's signed-rank test; $p<0.001 ; p=0.002 ; p=0.002 ; p=0.538)$. Thus, the majority of buildup neurons showed similar behavior in the Go-Go task, and this pattern was more evident when the trial conditions were presented in blocks.

Buildup neuron activity across the sample: a central cue affects stimulus interactions in the Go-Go task

When the cue located at the center of the screen appeared, monkeys were required to select one of the two stimuli as the saccade target. Consistent with a biased competition, when the target in the center location was selected (2T), we expected the level of neuronal activity to closely match the triangle-alone condition (1T). Similarly, when the square was indicated as the saccade target (2S), we expected the neuronal activity to closely match

\section{$\leftarrow$}

The rectangles at the bottom of each panel indicate the measurement intervals used for statistical comparisons, and filled rectangles indicate statistically significant differences across all conditions (ANOVA, $p<0.05$ ) (blue, visual interval; green, delay 1 interval; red, delay 2 interval; black, saccade interval). $\boldsymbol{b}$, Spike density function for a single neuron recorded in blocked trials of the Go-Go task. This example is an average of at least 20 trials. $c$, Spike density functions ( $\sigma=$ $15 \mathrm{~ms}$ ) averaged over 32 buildup neurons are shown for each of the four trial types in the blocked condition. 


\section{buildup neurons $1 \mathrm{~T}$ vs $2 \mathrm{~T}$}

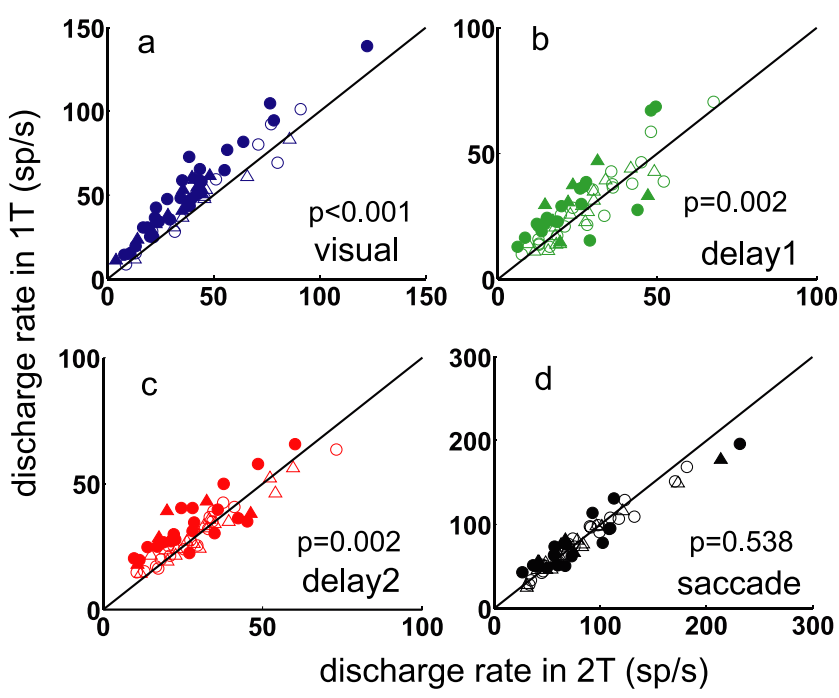

buildup neurons $2 \mathrm{~T}$ vs $2 \mathrm{~S}$

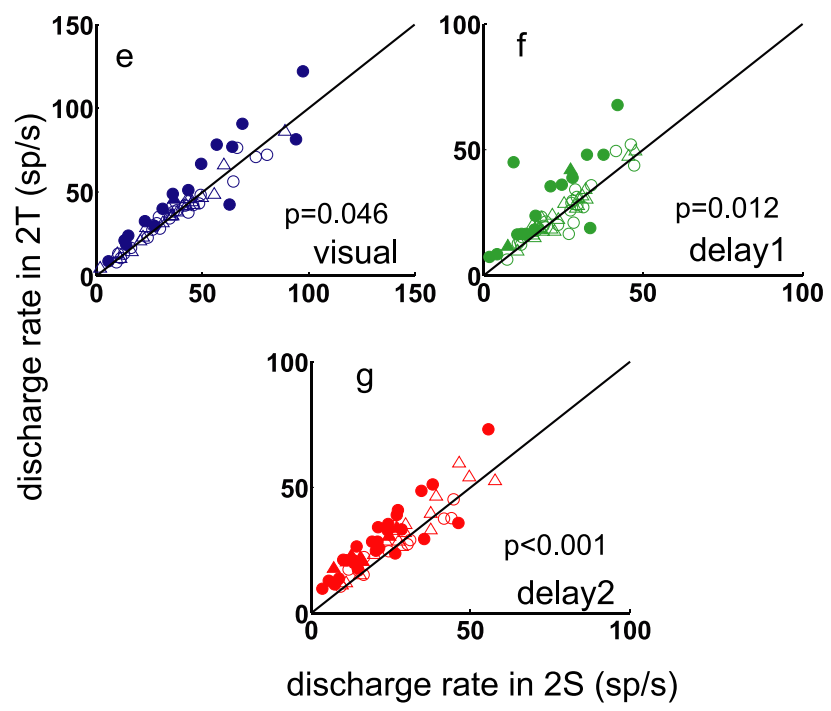

Figure 4. Buildup neurons have reduced activity when two stimuli are in the RF. The neuronal activity from individual buildup neurons ( 39 in blocked condition and 24 in the interleaved condition, 24 overlapping) in the $2 \mathrm{~T}$ condition is plotted against the neuronal activity in the $1 \mathrm{~T}$ condition. Points above the unity line indicate that the discharge rate in the $1 \mathrm{~T}$ condition was greater than the discharge rate in the $2 T$ condition. In $\boldsymbol{a}-\boldsymbol{d}$, the measurement interval plotted is indicated by the color, and the shape indicates trial condition. Circles are from blocked target trials, and triangles are from interleaved target trials. Blue circles and triangles are visual ( 0 $200 \mathrm{~ms}$ after stimulus onset), green circles and triangles are delay 1 (400 ms before the cue onset), red circles and triangles are delay $2(0-600 \mathrm{~ms}$ after cue onset), and black circles and triangles indicate the saccade interval ( $100 \mathrm{~ms}$ before to $100 \mathrm{~ms}$ after saccade onset). The filled symbols indicate that the differences between the conditions were statistically significant $(p<0.05)$. Open symbols indicate differences were not statistically significant. $\boldsymbol{e}-\boldsymbol{g}$, Neuronal activity measured in the $2 \mathrm{~T}$ condition was plotted against the neuronal activity measured in the 25 for the three intervals and the same neurons as in $\boldsymbol{a}$. The color and symbol schemes are the same as in $\boldsymbol{a}$. Each symbol is taken from a single neuron and is an average of 20-50 trials. Wilcoxon's signed-rank test was performed across the sample of neurons and was significant for each interval except the saccade interval indicated by the $p$ values.

that measured during the square-alone condition (1S). This pattern was seen in many buildup neurons (Fig. 3, middle and right panels). To determine whether this was consistent across our sample of neurons, we plotted the neuronal activity from the $2 \mathrm{~T}$ visual-tonic neurons - blocked - Go - Go task

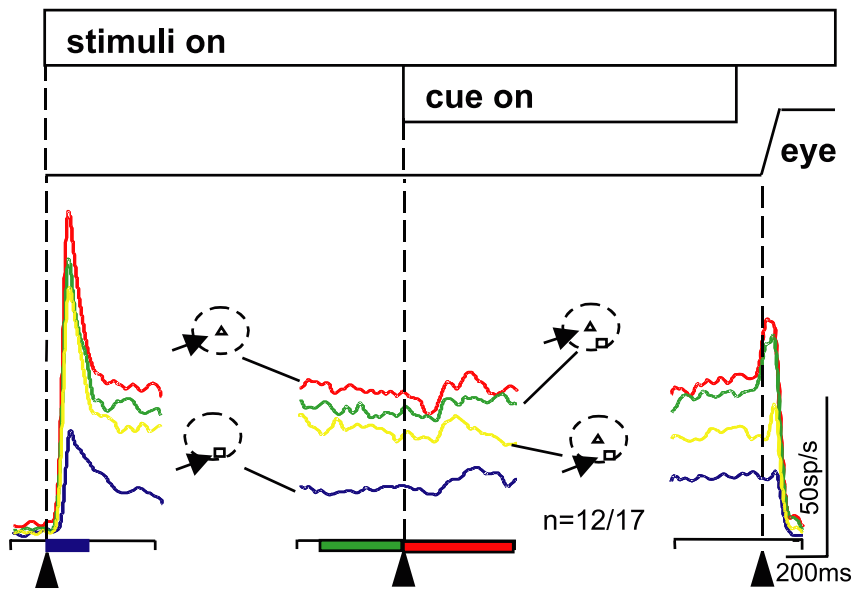

Figure 5. Visual-tonic neurons show reduced activity with two stimuli in the RF, and a central cue biases neuronal activity. The neuronal activity from 12 visual-tonic neurons is plotted as averaged, spike density functions ( $\sigma=15 \mathrm{~ms}$ ) for each of the four trial types. The arrangement and color scheme is identical to that shown in Figure 3. The data are taken from correctly performed trials when the conditions were blocked. Alignment times are indicated by the vertical dashed lines and the arrowheads at the bottom of each panel. Filled rectangles along the bottom of the panels indicate significant differences across the four conditions (ANOVA, $p<0.05$ ).

trials against the neuronal activity in the $2 \mathrm{~S}$ trials for the visual, delay 1 and delay 2 measurement intervals (Fig. $4 e-g$ ). In the blocked target trials, we found that 14 of 39 (36\%) buildup neurons had significantly ( $t$ test, Bonferroni corrected, $p<0.05$ ) higher visual responses for the $2 \mathrm{~T}$ condition compared with the $2 \mathrm{~S}$ condition (Fig. $4 e$, filled blue circles, points above the unity line) in the visual interval. Fourteen of $39(36 \%)$ showed significantly higher activity measured during delay 1 (Fig. $4 f$, filled green circles), and 24 of 39 (62\%) buildup neurons had significantly greater activity for $2 \mathrm{~T}$ compared with $2 \mathrm{~S}$ during delay 2 (Fig. $4 g$, filled red circles) ( $t$ test, Bonferroni corrected, $p<0.05$ ). Because the monkeys could not know which stimulus would be identified as a saccade target in the interleaved conditions, we found few neurons with these differences before the saccade cue was provided. One of 24 and 2 of 24 showed significant differences during the visual and delay 1 intervals (Fig. 4e, filled blue triangles, $f$, filled green triangles), whereas 7 of 24 (29\%) showed significant differences during the delay 2 interval (Fig. $4 g$, filled red triangles) ( $t$ test, Bonferroni corrected, $p<0.05$ ). Consistent with a biased competition, when a cue indicated a saccade to a strong stimulus, suppressive stimulus interactions decreased. When a cue indicated a saccade to a weak stimulus (at the edge of the RF), the reduction in stimulus interactions was less obvious. Across all neurons in the sample, the differences were significant for the three intervals (Wilcoxon's signed-rank test; $p=0.046$; $p=0.012 ; p<0.001)$.

\section{Go-Go task: behavior of visual-tonic neurons}

We recorded from 17 visual-tonic neurons in the blocked trials, and nine of these were also recorded in the interleaved trials. We found that visual-tonic neurons behaved similarly to buildup neurons. Figure 5 shows the average neuronal activity profile recorded from 12 visual-tonic neurons (recall that we plotted spike density functions for all neurons excluding those showing opposite effects in at least one interval of the task). The initial visual response of these neurons was larger with a single stimulus in the RF compared with the condition in which a single stimulus 
visual-tonic neurons $1 \mathrm{~T}$ vs $2 \mathrm{~T}$
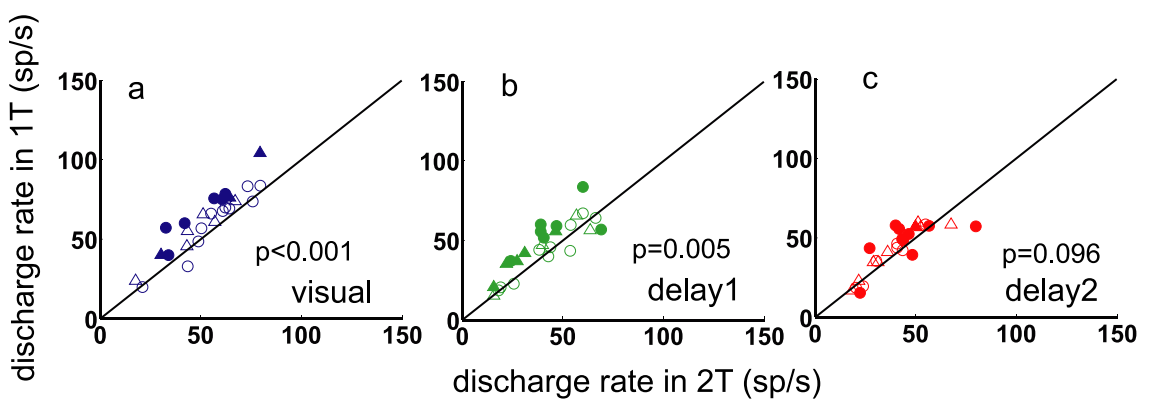

visual-tonic neurons $2 T$ vs $2 S$
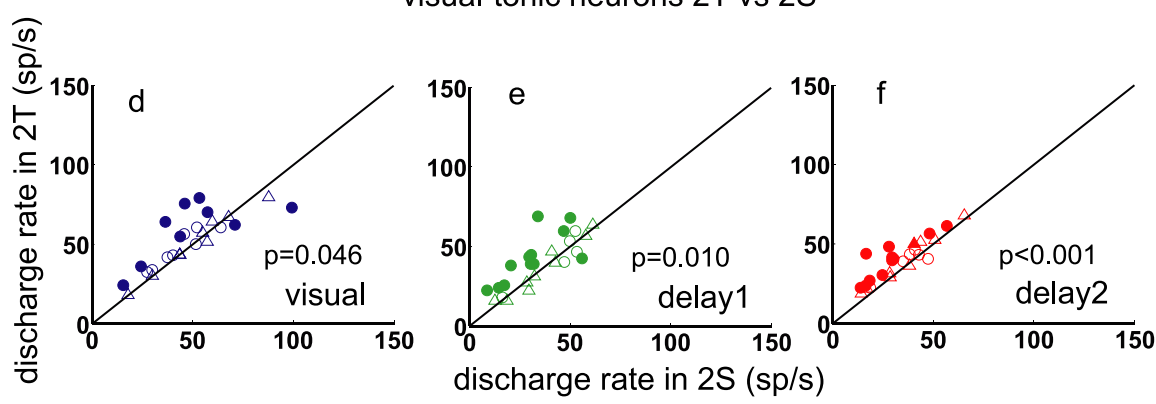

Figure 6. Visual-tonic neurons show reduced activity with two stimuli in RF, and a central cue biases neuronal activity. In $\boldsymbol{a}-\boldsymbol{c}$, the neuronal activity from individual visual-tonic neurons (17 blocked and 9 interleaved) in the 1T condition is plotted against the activity measured in the $2 \mathrm{~T}$ condition. Points above the unity line indicate that the discharge rate in the $1 \mathrm{~T}$ condition was greater than the $2 T$ condition. Intervals are indicated by color, and blocked or interleaved trial types are indicated by shape. Filled symbols indicate that differences were statistically significant. The arrangement is the same as in Figure 4, except the saccade interval is not shown. In $\boldsymbol{e}-\boldsymbol{g}$, the neuronal activity in the $2 \mathrm{~T}$ condition was plotted against the neuronal activity measured in the $2 \mathrm{~S}$ condition. Each symbol is taken from a single neuron and is the mean of at least 20-50 trials. Wilcoxon's signed-rank test was performed across all neurons and was significant for each interval except the delay 2 interval in the 1T versus 2T comparison as indicated by the $p$ values.

was located at the edge of the RF (Fig. 5, red and blue traces). The activity was also greater for a single stimulus compared with when two stimuli were present in the RF (Fig. 5, red, green, and yellow traces).

Visual-tonic activity across the sample: two stimulus interactions In blocked trials, 6 of 17, 6 of 17, and 7 of 17 of visual-tonic neurons had significantly ( $t$ test, $p<0.05$, Bonferroni corrected) lower activity in $2 \mathrm{~T}$ compared with $1 \mathrm{~T}$ target trials in all three measurement intervals (Fig. $6 a-c$, filled blue, green, and red circles). In interleaved trials, three of nine (33\%) neurons had a significantly lower level of activity in the $2 \mathrm{~T}$ condition compared with the $1 \mathrm{~T}$ condition in the visual interval (Fig. $6 a$, filled blue triangles) ( $t$ test, Bonferroni corrected, $p<0.05)$. Five of nine $(55 \%)$ had less activity in the $2 \mathrm{~T}$ condition compared with the $1 \mathrm{~T}$ condition during delay 1 (Fig. $7 b$, filled green triangles), and one of nine (11\%) had less activity during the delay 2 interval (Fig. $6 c$, filled red triangles) ( $t$ test, Bonferroni corrected, $p<0.05$ ). Across all neurons in the sample, the differences were significant for the visual and delay 1 intervals but not the delay 2 interval (Wilcoxon's signed-rank test; $p<0.001 ; p=0.005 ; p=0.096$ ).

Visual-tonic activity across the sample: a central cue affects stimulus interactions in the Go-Go task

As we had done for buildup neurons, we plotted visual-tonic neuronal activity in the $2 \mathrm{~T}$ condition against that measured in the $2 \mathrm{~S}$ condition for each measurement interval (Fig. $6 d-f$ ). We found that, in blocked trials, 7 of 17 (41\%) visual-tonic neurons showed significantly higher visual activity when monkeys knew they should select a saccade target located in the center of the RF compared with when they knew they should select a saccade target located at the edge of the RF (Fig. $6 d$, filled blue circles, blocked; filled blue triangles, interleaved) ( $t$ test, Bonferroni corrected, $p<0.05$ ). This was also evident for delay 1 intervals (Fig. 6e, filled green circles, blocked; filled green triangles, interleaved) and delay 2 intervals (Fig. $6 f$, filled red circles, blocked; filled red triangles, interleaved) ( $t$ test, Bonferroni corrected, $p<0.05$ ). Across all neurons in the sample, the differences were significant for all three intervals (Wilcoxon's signed-rank test; $p=0.046 ; p=$ $0.010 ; p<0.001)$.

\section{Go/No-Go task: behavior of buildup neurons}

In extrastriate cortex, sensory interactions and the ability of attention to bias these interactions were tested by comparing attended conditions with unattended conditions (Reynolds et al., 1999). We sought to dissociate stimulus interactions from processes related to events preceding eye movement initiation. Therefore, we recorded from buildup and visual-tonic neurons while monkeys performed the Go/No-Go task. In our Go/No-Go task, when the fixation point changed shape to a triangle, this indicated that a saccade to the triangle would be required. When the fixation point changed shape to a square, this indicated that the monkey should hold its gaze at the fixation point. In the latter condition, we could examine stimulus interactions while the monkeys remained fixating and presumably did not engage in processes leading up to a saccade. We assumed this was analogous to the unattended condition in previous experiments. We measured neuronal activity in two variations of this scheme. In one, the triangle was located in the center of the RF and was always associated with the production of a saccade. This stimulus was "strong" because it drove the neuron maximally. The square located at the edge of the RF drove the neuron less well and therefore was similar to the introduction of a weak stimulus. Therefore, using this visual stimulus configuration, we could assess the influence of cueing a gaze shift to a strong stimulus on the stimulus interactions. In the second variation, the triangle was located at the edge of the RF. This stimulus was still excitatory for the neuron, but it drove the neuron less well. The square was now located at the center of the RF and drove the neuron maximally. In this arrangement, we could assess the influence of a cue to shift gaze to a weak (edge) stimulus on the stimulus interactions.

First, we describe the results from buildup neurons in the experiments in which the influence of a strong stimulus was assessed (Figs. 7, 8), and we describe results from buildup neurons in the experiments in which the influence of a weak stimulus was assessed (Fig. 9). Second, we describe the results from visualtonic neurons in the same two stimulus configurations (Figs. 10, 11).

We recorded from 60 buildup neurons in the Go/No-Go task. Forty-four neurons were recorded with the trial conditions randomly interleaved, and 47 neurons were recorded with the trial 
buildup neurons - blocked - Go/No - Go task

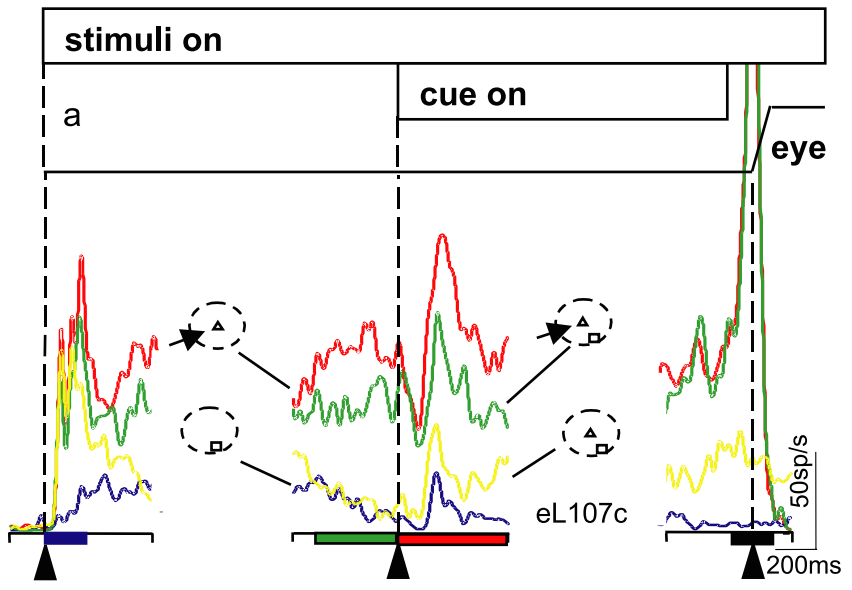

buildup neurons - blocked - Go/No - Go task

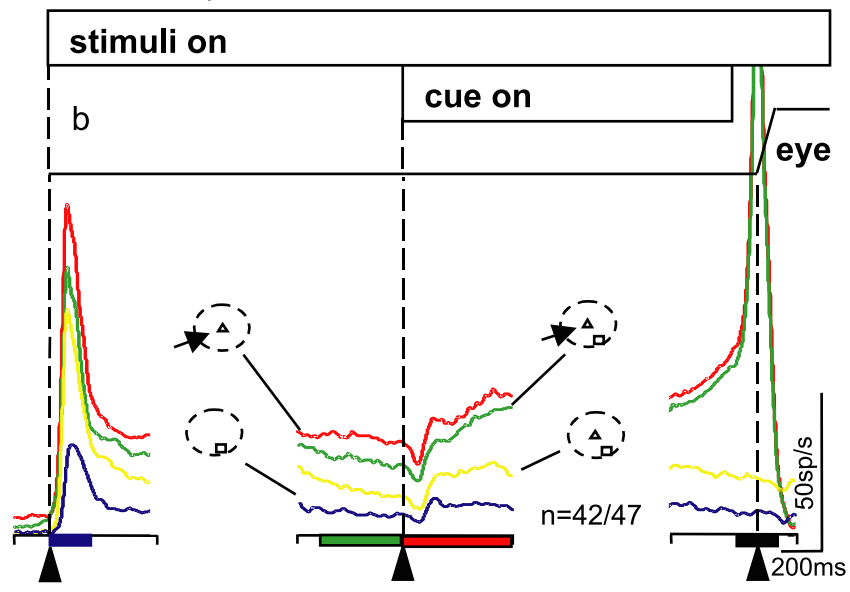

Figure 7. Cueing a strong stimulus influences buildup neuron activity. The temporal arrangement of the task is shown by the horizontal bars along the top, and the line labeled "eye" is a schematic of eye position. The panel on the left is aligned on the onset of the stimuli indicated by the vertical dashed line and arrowhead. The middle panel is aligned on the onset of the cue, and the right panel is aligned on the onset of the saccade also indicated by the vertical dashed line and the arrowhead. The arrangement and color scheme are the same as in Figure 3 , $\boldsymbol{b}$ and $\boldsymbol{c}$. $\boldsymbol{a}$, Single neuron example recorded in the Go/No-Go task. $\boldsymbol{b}$, Mean spike density function from 42 buildup neurons. Filled rectangles along the bottom of the panels indicate significant differences across the four conditions (ANOVA, $p<0.05$ ).

conditions blocked. Thirty-one of these neurons were recorded in both the interleaved and blocked conditions (Table 1).

Influence of cueing a strong stimulus in buildup neurons

In Figure 7, we show a single neuron example (Fig. $7 a$ ) and an average of 42 buildup neurons (Fig. $7 b$ ) recorded in the blocked version of the Go/No-Go task. We found three differences in the neuronal activity in this task compared with the Go-Go task (Figs. $3 c, 7 b$ ). First, immediately before the saccade interval, the level of activity in the $2 \mathrm{~T}$ condition matched the level of activity in the 1T condition more closely in the Go-Go task than in the Go/No-Go task (Figs. $3 c, 7 b$, right panels, green and red traces). Second, immediately before the saccade interval, the neuronal activity in the $2 S$ condition matched the neuronal activity in the $1 \mathrm{~S}$ condition more closely in the Go-Go task than in the Go/No-Go task (Figs. $3 c, 7 b$, right panels, yellow and blue traces). Third, and expectedly, there was no saccade-related burst in the $2 \mathrm{~S}$ condition in the Go/No-Go task, although there was a saccaderelated burst in the $2 \mathrm{~S}$ condition of the Go-Go task because a

\section{buildup neurons $1 \mathrm{~T}$ vs $2 \mathrm{~T}$}

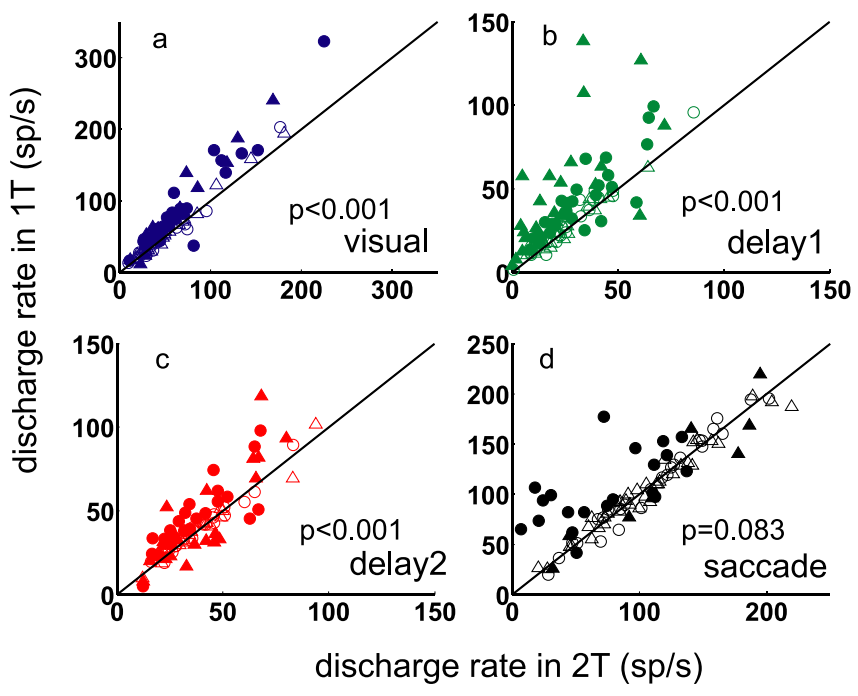

buildup neurons $2 \mathrm{~T}$ vs $2 \mathrm{~S}$

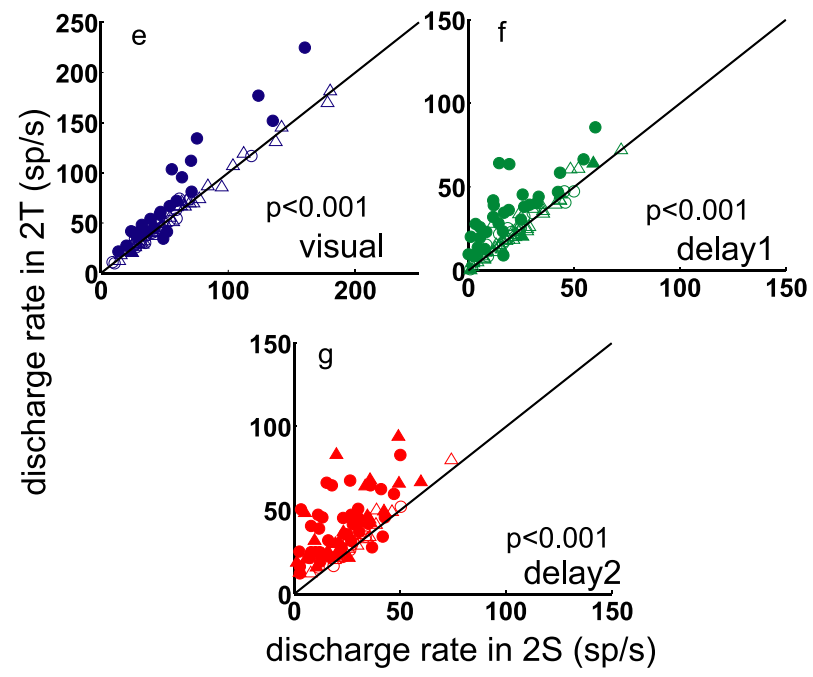

Figure 8. Cueing a strong stimulus influences most buildup neurons. Neuronal activity in the $1 \mathrm{~T}$ condition is plotted against the neuronal activity in the $2 \mathrm{~T}$ condition for buildup neurons (47 blocked and 44 interleaved). $\boldsymbol{a}-\boldsymbol{d}$ show the different intervals, visual, delay 1 , delay 2 , and saccade. In $\boldsymbol{e}-\boldsymbol{g}$, the neuronal activity measured in the $2 \mathrm{~T}$ condition was plotted against the neuronal activity measured in the 25 condition for the visual, delay 1 , and delay 2 intervals. Each symbol is taken from a single neuron and is the mean of at least $20-50$ trials. The arrangement and color scheme of this figure is the same as that shown in Figure 4. Circles are from blocked trials, and triangles are from interleaved trials. The filled symbols indicate that the differences between the conditions were statistically significant $(p<0.05)$. Open symbols indicate differences were not statistically significant. Blue circles and triangles are data from the visual interval $(0-200 \mathrm{~ms}$ after stimulus onset). Green circles and triangles are data from the delay 1 interval ( $400 \mathrm{~ms}$ before cue onset). Red circles and triangles are data from the delay 2 interval $(0-600$ $\mathrm{ms}$ after cue onset). Black circles and triangles are data from the saccade interval ( $100 \mathrm{~ms}$ before to $100 \mathrm{~ms}$ after saccade onset). Wilcoxon's signed-rank test was performed across the sample of neurons and was significant for all intervals except the saccade interval.

saccade was made only in the latter task (Figs. $3 c, 7 b$, right panels, yellow traces).

As we observed for the Go-Go task, in the Go/No-Go task, when two stimuli were presented in the same RF of buildup neurons, the activity was lower than when a single stimulus was presented during the visual, delay 1 , and delay 2 intervals (Fig. $8 a-c$, blue, green, and red circles, blocked; blue, green, and red trian- 
buildup neurons - blocked - Go/No - Go task

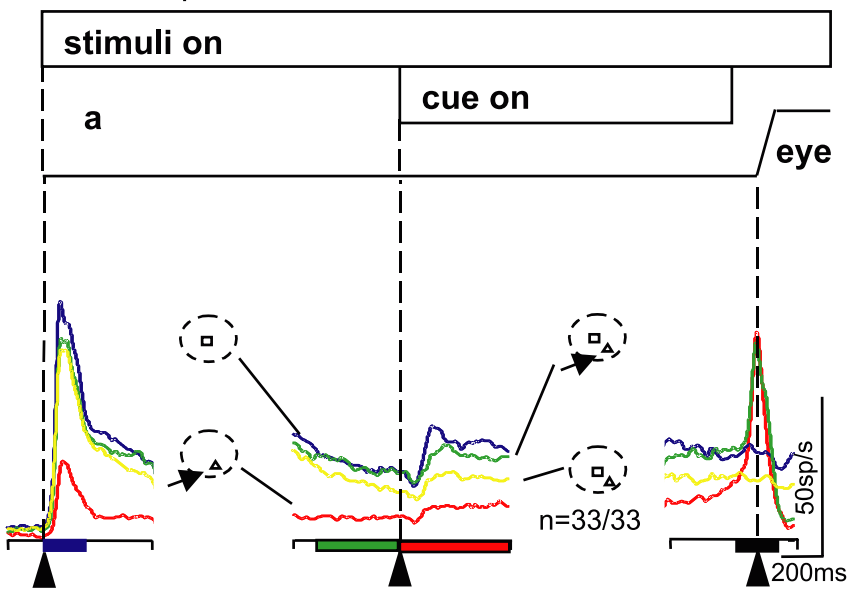

buildup neurons $2 \mathrm{~T}$ vs $2 \mathrm{~S}$

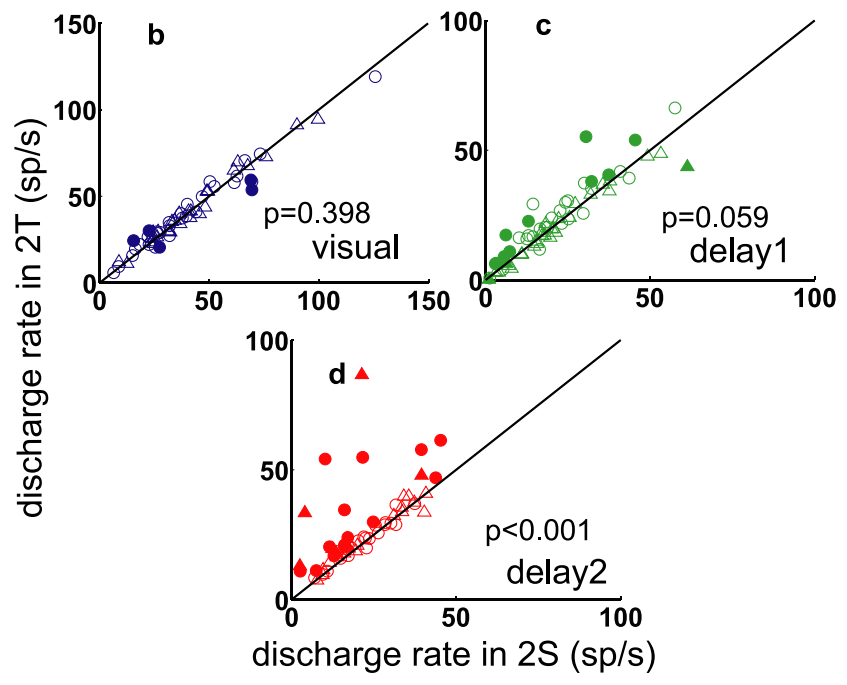

Figure 9. Cueing a weak stimulus influences buildup neuron activity. $\boldsymbol{a}$, The temporal arrangement of the task is shown by the horizontal, open bars at the top. The line labeled "eye" is a schematic of eye position. The alignment of each panel is indicated by the vertical dashed lines and the arrowheads. The left panel is aligned on stimuli onset. The middle panel is aligned on the cue onset, and the right panel is aligned on saccade onset. Each trace is an average of 33 buildup neurons. The spike density functions ( $\sigma=15 \mathrm{~ms}$ ) are superimposed for the different stimulus conditions. Red traces are from $1 \mathrm{~T}$ trials, blue traces are from $1 S$ trials, green traces are from $2 \mathrm{~T}$ trials, and yellow traces are from $2 S$ trials. Note that, in this version of the task, the triangle stimulus was placed at the edge of the RF, whereas the square ( $\left.N 0-\mathrm{G}_{0}\right)$ stimulus was placed at the center of the RF. The filled rectangles along the bottom of each panel indicate the measurement intervals for which the conditions were significantly different (ANOVA $p<0.05$ ). $\boldsymbol{b}$ - $\boldsymbol{d}$, The neuronal activity measured in the $2 \mathrm{~T}$ condition was plotted against the neuronal activity measured in the 25 condition for buildup neurons ( 33 blocked and 29 interleaved, 29 in both blocked and interleaved). $\boldsymbol{b}$, Visual; $\boldsymbol{c}$, delay $1 ; \boldsymbol{d}$, delay 2 . Each symbol is taken from a single neuron and is the mean of at least $20-50$ trials. Points above the unity line indicate that the neuronal activity in the $2 \mathrm{~T}$ condition was greater than the $2 \mathrm{~S}$ condition. The arrangement is the same as in Figure $8 e-g$.

gles, interleaved) ( $t$ test, Bonferroni corrected, $p<0.05)$. At the time the cue was provided, indicating that a saccade should be prepared in the case of the triangle cue, or that the monkey should remain fixating in the case of the square cue, the neuronal activity changed. For the sample of 47 neurons recorded in the blocked condition, 23 of $47(49 \%)$ had a significantly ( $t$ test, $p<0.05$, Bonferroni corrected) higher level of activity in the $2 \mathrm{~T}$ condition visual-tonic neurons - blocked - Go/No - Go task

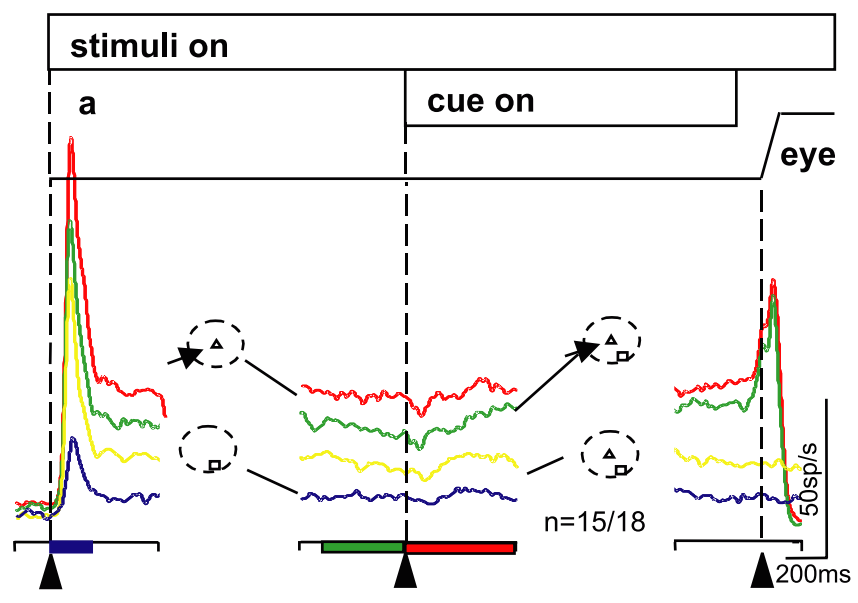

visual-tonic neurons $2 T$ vs $2 S$

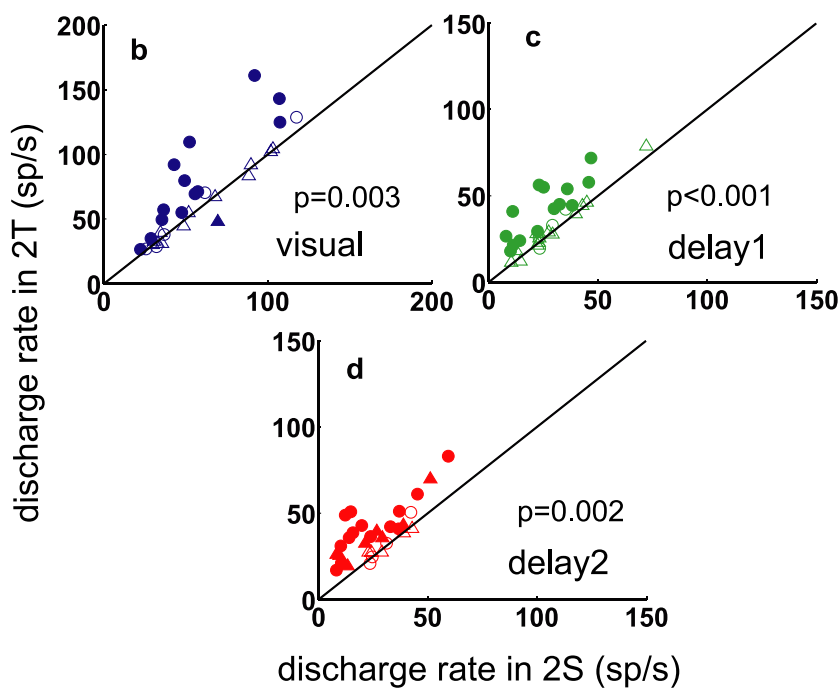

Figure 10. Cueing a strong stimulus influences visual-tonic neuron activity. $\boldsymbol{a}$, The horizontal bars at the top of the figure show the temporal arrangement of the task. The line labeled "eye" is a schematic of eye position. The vertical dashed lines and the arrowheads in each panel indicate the alignment time. The left panel is aligned on stimuli onset, the middle panel is aligned on cue onset, and the right panel is aligned on saccade onset. The color scheme is the same as in Figure 3. Red traces are from $1 \mathrm{~T}$ trials, blue traces are from $1 \mathrm{~S}$ trials, green traces are from $2 T$ trials, and yellow traces are from $2 S$ trials. The rectangular bars along the bottom of each panel indicate the measurement intervals and, if filled, indicate that there were significant differences $(p<0.05)$ between all four conditions. $\boldsymbol{b}-\boldsymbol{d}$, The neuronal activity measured in the $2 \mathrm{~T}$ condition was plotted against the neuronal activity measured in the $2 S$ condition. Each symbol is taken from a single neuron and is the mean of at least $20-50$ trials. The arrangement and color scheme of this figure is the same as that shown in Figure 9 . Circles are from blocked target trials, and triangles are from interleaved target trials. The filled symbols indicate that the differences between the conditions were statistically significant $(p<0.05)$. Open symbols indicate that differences were not statistically significant. Wilcoxon's signed-rank test was performed across the sample, and the differences were significant for all intervals.

compared with the $2 S$ condition during the visual interval (Fig. $8 e$, blue circles, blocked; blue triangles, interleaved). Twentyeight of $47(60 \%)$ showed this enhancement in the delay 1 interval (Fig. 8f, green circles, blocked; green triangles, interleaved) ( $t$ test, Bonferroni corrected, $p<0.05)$. Thirty-six of $47(77 \%)$ showed the enhancement during the delay 2 interval (Fig. $8 g$, red circles, blocked; red triangles, interleaved) ( $t$ test, Bonferroni corrected, $p<0.05$ ). Thus, a cue indicating a shift of gaze to a strong stimulus reduced the influence of a weak stimulus on SC buildup 


\section{visual-tonic neurons - blocked - Go/No - Go task}

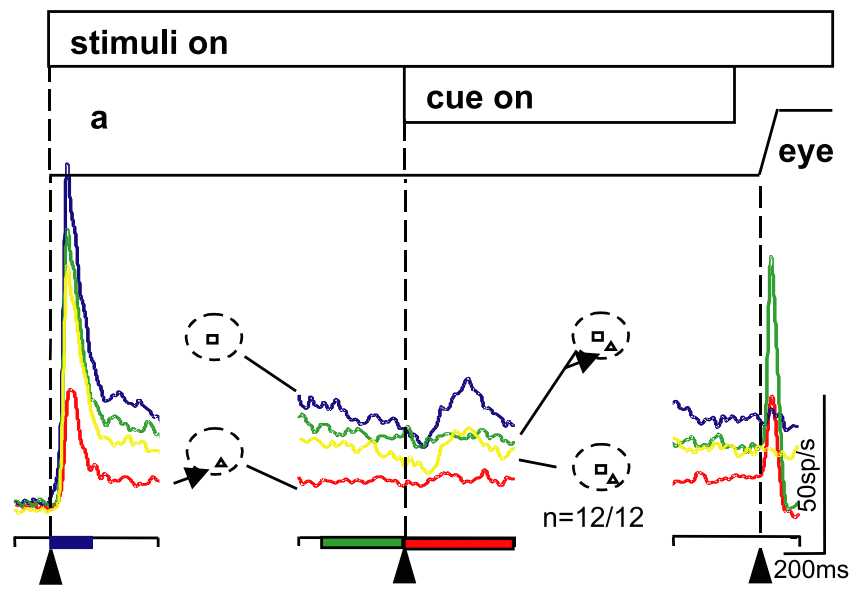

visual-tonic neurons $2 T$ vs $2 S$

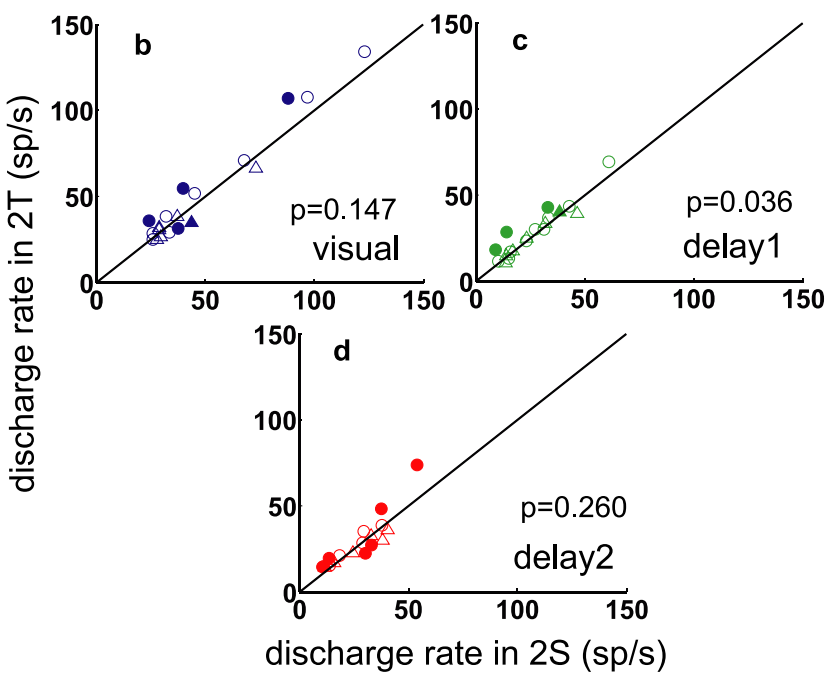

Figure 11. Cueing a weak stimulus influences visual-tonic neuron activity. $\boldsymbol{a}$, The temporal arrangement of the task is shown by the horizontal, open bars at the top of the figure. The line labeled "eye" is a schematic of eye position. The alignment of each panel is indicated by the vertical dashed lines and the arrowheads. The left panel is aligned on stimuli onset. The middle panel is aligned on the cue onset, and the right panel is aligned on saccade onset. Each trace is an average of 12 visual-tonic neurons. The spike density functions ( $\sigma=15 \mathrm{~ms}$ ) are superimposed for the different stimulus conditions. Red traces are from $1 \mathrm{~T}$ trials, blue traces are from $1 \mathrm{~S}$ trials, green traces are from $2 \mathrm{~T}$ trials, and yellow traces are from $2 \mathrm{~S}$ trials. Note that, in this version of the task, the triangle stimulus was placed at the edge of the RF, and the square (No-Go) stimulus was placed at the center of the RF. $\boldsymbol{b}-\boldsymbol{d}$, The neuronal activity measured in the $2 T$ condition was plotted against the neuronal activity measured in the $2 S$ condition for the visual-tonic neurons ( 12 blocked and 7 interleaved). Each symbol is taken from a single neuron and is the mean of at least 20-50 trials. Circles are from blocked target trials, and triangles are from interleaved target trials. The filled symbols indicate that the differences between the conditions were statistically significant $(p<0.05)$. Open symbols indicate that differences were not statistically significant. Blue circles and triangles are data from visual interval (0-200 ms after stimulus onset), green circles and triangles are data from the delay 1 interval (400 ms before cue onset), and red circles and triangles are data from the delay 2 interval (0 - 600 ms after cue onset). Wilcoxon's signed-rank test across the sample indicated that only the delay 2 interval differences were significant.

neurons. Over the entire sample, we found significant differences in all of the intervals except the saccade intervals (Wilcoxon's test; $p<0.001$, Fig. $8 a$; $p<0.001$, Fig. $8 b ; p<0.001$, Fig. $8 c$; $p=0.083$, Fig. $8 d$; $p<0.001$, Fig. $8 e ; p<0.001$, Fig. $8 f ; p<0.001$, Fig. $8 g$ ).
Influence of cueing a weak stimulus in buildup neurons In these experiments, the target associated with a saccade (triangle) was presented at the edge of the RF. The No-Go target (square) was presented in the center of the RF, making it a strong stimulus for the neuron. Accordingly, the biased competition model applied to the SC predicts that a cue to shift gaze to the edge or "weak" stimulus should reduce the influence of the stimulus located in the center of the RF. Therefore, the activity levels in the $2 \mathrm{~T}$ and $2 \mathrm{~S}$ conditions should be much closer than what was observed in Figure 7.

Figure 9 shows the average response profile of the 33 buildup neurons recorded in this version of the task. The same neurons were recorded in the previous configuration. During all three intervals, the neuronal activity in the $2 \mathrm{~T}$ and $2 \mathrm{~S}$ trial types were much closer than that seen in the $2 \mathrm{~T}$ and $2 \mathrm{~S}$ condition with the opposite stimulus configuration (Fig. 9, left side of middle panel, blue, green and yellow traces). Note that, for the same data in this condition, we are interested in the values that were not significantly different or were lower in the $2 \mathrm{~T}$ compared with the $2 \mathrm{~S}$ condition. In the blocked condition, 31 of 33 (94\%) buildup neurons had the same or lower activity in the $2 \mathrm{~T}$ condition compared with the $2 \mathrm{~S}$ condition during the visual interval (Fig. $9 b$, open and filled blue circles, blocked; open and filled blue triangles, interleaved). Twenty-three of 33 (67\%) showed the same or lower activity during the delay 1 interval (Fig. $9 c$, open and filled green circles, blocked; open and filled green triangles, interleaved). In the delay 2 interval, 20 of 33 (61\%) buildup neurons had the same or lower activity for the $2 \mathrm{~T}$ trials compared with the $2 S$ trials (Fig. $9 d$, open and filled red circles, blocked; open and filled red triangles, interleaved). Note also that the $1 \mathrm{~S}$ condition was high at the time of the cue because there continued to be a stimulus in the center of the RF. Thus, cueing a weak stimulus, although it was still within the excitatory region of the SC neuron, did not produce the same enhancement as seen when a strong stimulus was cued. We will explore this phenomenon more thoroughly in the section below (see Direct comparison with the biased competition model).

\section{Go/No-Go task: behavior of visual-tonic neurons}

We recorded from 20 visual-tonic neurons in the Go/No-Go task (Fig. 10). Thirteen were recorded in interleaved trials, and 18 were recorded in blocked trials. Eleven were recorded in both types (Table 1). The results were qualitatively identical to those seen in buildup neurons.

\section{Influence of cueing a strong stimulus in visual-tonic neurons}

Again, in this condition, we predicted that, if a cue to shift gaze resulted in neuronal activity that biased the competition between locations, then $2 \mathrm{~T}$ activity should be higher than $2 \mathrm{~S}$ activity. Across our sample of visual-tonic neurons, respectively, 13 of 18 (72\%) and 14 of 18 (78\%) had significantly higher activity for the $2 \mathrm{~T}$ condition during the visual and delay 1 intervals in the blocked condition (Fig. 10b,c, filled blue and green circles, blocked; filled blue and green triangles, interleaved) ( $t$ test, Bonferroni corrected, $p<0.05$ ). When monkeys were provided with a cue indicating that they should prepare a saccade (delay 2), 14 of $18(78 \%)$ visual-tonic neurons in the blocked condition (Fig. 10d, filled red circles, blocked; filled red triangles, interleaved) had a significantly ( $t$ test, Bonferroni corrected, $p<0.05$ ) higher activity for $2 \mathrm{~T}$ compared with $2 \mathrm{~S}$. Across the sample, significant differences were found in all three intervals (Wilcoxon's signedrank test; $p=0.003 ; p<0.001 ; p=0.002)$. 
Influence of cueing a weak stimulus in visual-tonic neurons Recall that, in this condition, we predicted that, if a cue to shift gaze to the edge (weak) stimulus resulted in neuronal activity that biased the competition between locations, then $2 \mathrm{~T}$ activity should be the same or lower than $2 \mathrm{~S}$ activity. We recorded from 12 visual-tonic neurons in this version of the task (Fig. 11). These 12 neurons were also recorded in the opposite configuration. Many visual-tonic neurons showed no significant difference for $2 \mathrm{~T}$ compared with the $1 \mathrm{~T}$ condition (Fig. $11 b-d$, open blue, green, and red circles, blocked; open blue, green, and red triangles, interleaved). Across the sample, only delay 1 showed significant differences (Wilcoxon's signed-rank test; $p=0.147 ; p=$ $0.036 ; p=0.260)$.

\section{Direct comparison with the biased competition model}

To provide an explicit test of whether the biased competition model explained the results we obtained in SC, we adopted an analysis that was used to assess V2 and V4 neurons (Reynolds et al., 1999). A selectivity index was compared with a sensory interaction index. The values of both indices ranged from -1 to 1 . In extrastriate cortex, stimuli were arbitrarily defined as reference or probe stimuli. If a neuron had a negative selectivity index, the neuron had a smaller response to a probe stimulus compared with a reference stimulus. If a neuron had a positive selectivity index, the neuron had a larger response to the probe than to the reference. The sensory interaction index determined how the neurons behaved in the presence of the two stimuli together. A negative interaction index indicated that the neuronal response to the pair was dominated by the neuronal response to reference stimulus, whereas a positive interaction index meant that the pair response was dominated by the probe stimulus. The slope of the line relating the sensory interaction and selectivity indices indicated which stimulus dominated the pair response. In the feature attention experiments (Reynolds et al., 1999), comparing the slope of these two variables with and without attention provided a way to assess the influence of attention. When attention was directed to the probe stimulus, the response of the probe dominated the pair response and the slope increased. When attention was directed to the reference stimulus, the probe had a reduced influence on the pair response and the slope was reduced. Thus, attention acted on the underlying sensory interactions (Reynolds et al., 1999, their Figs. 4, 10, 11).

To determine whether a cue to shift gaze also acted on sensory stimulus interactions within the SC, we computed a sensory interaction index and plotted this against a selectivity index (Fig. 12). We used 32 neurons (25 buildup and 7 visual-tonic) recorded in both configurations of the task in the interleaved trial types. We measured the neuronal activity for the neurons when both stimuli were present (pair) and when only a single stimulus was present in the center of the RF (reference). We then measured the sum of these two activities. The ratio of the pair - reference activity to the pair + reference activities was our measure of the sensory interaction. Negative values indicate that the response of the neuron is suppressed in the pair condition. Positive values indicate that the response of the neuron is enhanced in the pair condition. Importantly, the interaction index was computed during the interval before the presentation of the centrally located cue indicating whether or not a saccade would be required (delay 1) and after the cue (delay 2). This measure was computed for the two visual stimulus configurations of the Go/No-Go task. The configuration in which the triangle (Go cue) was located at the edge of the RF is shown in Figure 12, $a$ and $b$. The configuration in which the triangle (Go cue) was located in the center of the

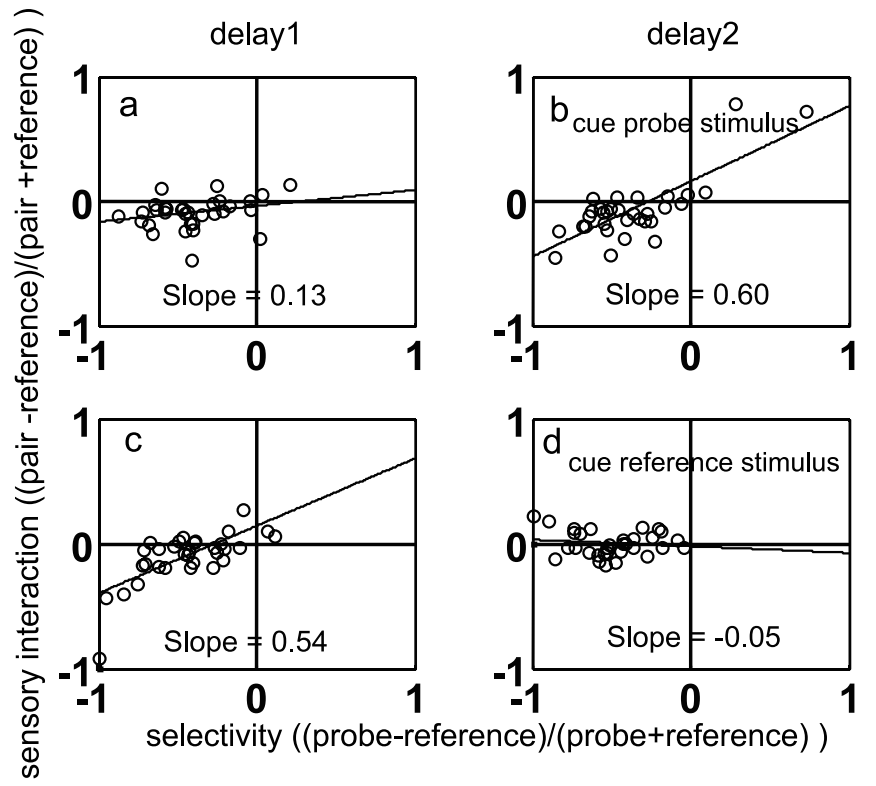

Figure 12. Cue to shift gaze influences sensory interactions in SC neurons. The sensory interaction index is plotted against the selectivity index for buildup $(n=25$ recorded in both the center and edge conditions in interleaved trials) and visual-tonic ( $n=7$ recorded in center and edge conditions in interleaved trials) neurons in the Go/No-Go task. The same neurons are displayed in all four panels. For the sensory interaction measure, the mean discharge rate of neurons during the presentation of two stimuli in a single RF was measured (pair), and the mean discharge rate of the same neurons when only a single stimulus was presented at the center (reference) of the RF was subtracted from the pair measure. We then divided this difference value by the sum of the same two quantities. For the selectivity index, we measured the mean discharge rate of the same neurons when a single stimulus was located at the edge (probe) of the RF and at the center of the RF (reference). The difference of these two quantities was divided by the sum of these two quantities. $\boldsymbol{a}$, Sensory interaction plotted against the selectivity index for delay 1 using the trials in which the center (reference) stimulus was the square (no saccade) and the edge (probe) stimulus was the triangle (saccade) during delay 1 , before the cue to make or withhold a saccade was provided. $\boldsymbol{b}$, The same data were plotted for the delay 2 interval after the cue to make a saccade to the edge stimulus was provided. $c$, The sensory interaction index is plotted against the selectivity index for the Go/No-Go trials in which the center (reference) stimulus was the triangle and the edge (probe) stimulus was the square. Delay 1 , before the cue, measurement interval was used. $\boldsymbol{d}$, The same as in $\boldsymbol{c}$, except for the delay 2 interval after the cue to make a saccade. Each circle is an average of at least 20-50 trials.

RF is shown in Figure 12, $c$ and $d$. In both cases, the center stimulus was defined as the reference stimulus, and the edge stimulus was defined as the probe. During delay 1 when the square was located in the center of the RF and the triangle was located at the edge of the RF, the slope of the sensory interaction versus the selectivity index was 0.13 (Fig. 12a). This indicated that the pair response was little influenced by the probe (the triangle on the edge of the RF). However, during delay 2, when the triangle was cued for a saccade to the edge of the RF, the slope increased to 0.60 . The influence of this cue was to pull the response of the pair toward a sensory interaction value that favored the probe stimulus response (the points fell more negative, resulting in an increased slope). Using multiple regression, we determined that the difference between these slopes was significant $(p<0.05)$.

In the Go/No-Go task in which the triangle was located in the center of the RF (reference) and the square was at the edge (probe), we computed the same indices. In this case, during delay 1 , the slope of the line was positive (0.54) (Fig. 12c). This was because the reference term in the sensory interaction index was larger in these trials even before the cue, because it was likely that the triangle would be cued for a saccade. After the cue to make a saccade to the reference stimulus, during delay 2 , the slope de- 
creased to -0.05 (Fig. 12d). This indicated that the cue to the reference stimulus changed the sensory interaction. The neuronal response became dominated by the reference stimulus (the points became less negative, resulting in a decrease in the slope). The difference in slopes in delay 1 and delay 2 were statistically significant $(p<0.05)$. Thus, we conclude that a cue to shift gaze, like attention in extrastriate cortex, influences stimulus interactions in SC neurons.

Two stimulus interactions: summation or averaging

By presenting two stimuli within single RFs of SC neurons, we could test whether a summation or an averaging model best explained the influence of multiple stimuli on SC neurons. In doing so, we could compare the behavior of SC neurons with the known behavior of neurons within the visual cortex (Ferrera and Lisberger, 1995, 1997; Treue and Maunsell, 1996; Recanzone et al., 1997; Britten and Heuer, 1999; Reynolds et al., 1999). For the summation model, we measured the discharge rate of neurons in the single stimulus cases separately (1T and $1 S$ ) and summed these two activities. We then measured the actual discharge rates in the $2 \mathrm{~T}$ condition and constructed plots of the predicted discharge rate against the actual discharge rate. This computation was performed across different intervals of the task so that we also could explore the dynamics (Fig. 13a-d). We performed the same computations for visual-tonic neurons (Fig. 14a-c). For the averaging model, we computed the predicted discharge rates by summing the neuronal activity for the $1 \mathrm{~S}$ and $1 \mathrm{~T}$ conditions and dividing by 2 . We then measured the actual discharge rates observed in the $2 \mathrm{~T}$ condition and plotted the predicted rate against the actual rate. Again, this was performed across multiple intervals (Fig. 13e-h) and was done also for visual-tonic neurons (Fig. 14d-f).

In the plots of predicted against actual discharge rates, if the model explained the data well, we expected to see the points fall along the line of unity, indicating that the two values were similar. In the visual interval, the summation model did not explain the observations in SC buildup neurons for either the Go-Go task or the Go/No-Go task (Fig. 13a, + and $\times$ symbols). Indeed, the summation model was a poor predictor of the neuronal activity for all of the intervals in both task conditions (Fig. 13a-d, red + and blue $\times$ symbols). The averaging model, in contrast, was much better (Fig. 13e-h). For the visual, delay 1, and delay 2 intervals, the neuronal activity in the two stimulus condition, in both the Go-Go and the Go/No-Go task, was very well explained by the average of the neuronal discharge seen in the single stimulus conditions (Fig. $13 e-g$, red + and blue $\times$ symbols). During the saccade interval, however, the pattern changed. Few of the points fell along the averaging line; rather, most fell below the line, indicating that the neuronal activity during this time was greater in the $2 \mathrm{~T}$ condition than would be predicted by averaging the two single stimulus condition responses (Fig. $13 h$, red + and buildup neurons

summation

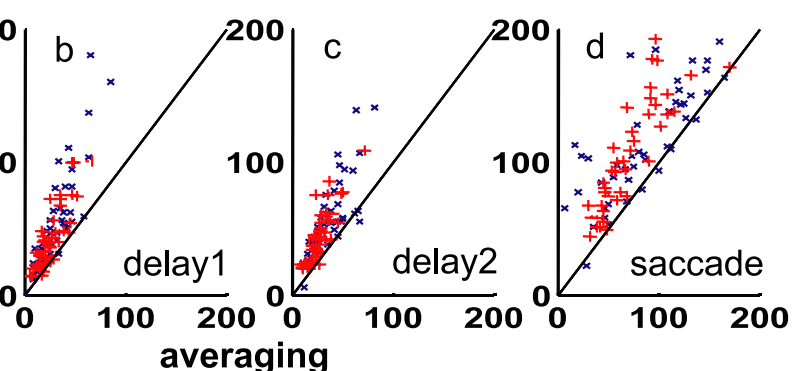

averaging

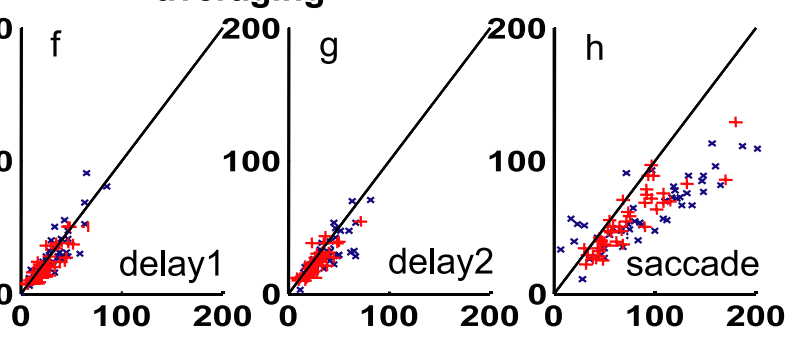

actual discharge rate in $2 \mathrm{~T}(\mathrm{sp} / \mathrm{s})$

+ Go-Go blocked $(\mathrm{n}=39)$

$X$ Go/No-Go blocked $(\mathrm{n}=47)$

Figure 13. Dynamics of multistimulus integration in buildup neurons. The predicted discharge rate of buildup neurons in the 2T condition if they summed the discharge rates from the $1 \mathrm{~T}$ and $1 \mathrm{~S}$ conditions is plotted against the actual discharge rates measured in the 2T condition. The data are taken from blocked target trials in which the monkeys performed the task correctly. $\boldsymbol{a}_{\text {, }}$ , conditions and dividing by 2 . The solid lines in all panels are the lines of unity. Points falling along this line indicate that the actual discharge rate was predicted accurately from the modeled discharge rate.

blue $\times$ symbols). Indeed, the activity measured in the $2 \mathrm{~T}$ condition was most similar to that seen in the $1 \mathrm{~T}$ condition during the saccade interval for both the Go-Go task (Fig. $4 d$ ) and the Go/ No-Go task (Fig. $8 d$ ). This is consistent with a winner-take-all readout at the time the saccade is initiated. Interestingly, this was more apparent for the neuronal activity measured in the Go/ No-Go task than the Go-Go task (Fig. 13h, red + and blue $\times$ symbols).

In visual-tonic neurons, the pattern was similar. For neither the Go-Go task nor the Go/No-Go task was the summation model satisfactory to explain the data (Fig. 13a-c, red + and blue $\times$ symbols). In contrast, the averaging model was better in all three intervals, visual, delay 1 , and delay 2 (Fig. $14 c-e$, red + and blue $X$ symbols). Interestingly, even the averaging model did not explain completely the results for these neurons in the Go-Go task, particularly during the visual interval (Fig. 14a, red +).

\section{Distribution of saccade parameters}

Because the stimuli in our task were very close to one another, often within $5^{\circ}$, it was possible that the modulations we observed between the $1 \mathrm{~T}$ and $2 \mathrm{~T}$ conditions indicated changes in saccade parameters, such as accuracy, precision, or velocity. To test this, we measured the endpoint and velocity of saccades in the $1 \mathrm{~T}$ and the $2 \mathrm{~T}$ conditions of the Go/No-Go task. We computed the mean and SD of the endpoints and velocities from 16 experiments in which the neuronal activity in the $1 \mathrm{~T}$ condition was significantly greater than in the $2 \mathrm{~T}$ condition during the saccade interval (Fig. $8 d$, filled black circle). We compared the vertical and horizontal 
visual-tonic neurons

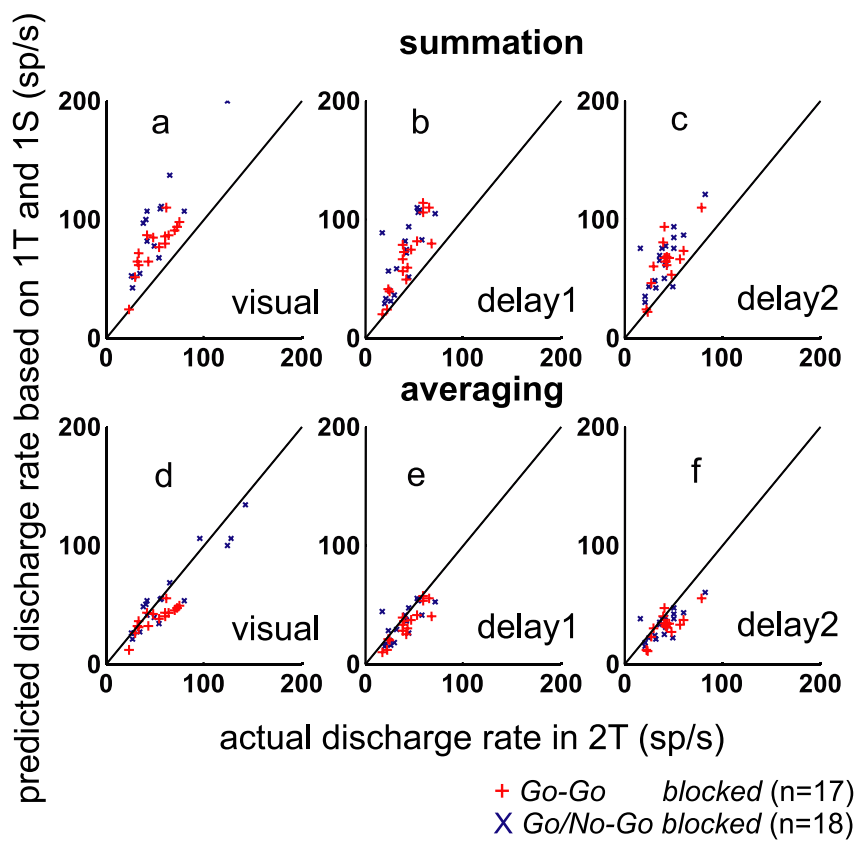

Figure 14. Dynamics of multistimulus integration in visual-tonic neurons. The predicted discharge rate of visual-tonic neurons in the $2 \mathrm{~T}$ condition if they summed the discharge rates from the $1 \mathrm{~T}$ and $1 \mathrm{~S}$ conditions is plotted against the actual discharge rates measured in the $2 \mathrm{~T}$ condition. The data are taken from blocked target trials in which the monkeys performed the task correctly. $\boldsymbol{a}$ - $\boldsymbol{c}$ show the predictions for each of the measurement intervals, visual, delay 1 , and delay 2. Red + are data from the $\mathrm{G} 0$-Go task, and blue $\times$ are taken from the $\mathrm{Go} / \mathrm{No}_{0}-\mathrm{Go}_{0}$ task trials. $\boldsymbol{e}-\boldsymbol{g}$ are the same data and intervals plotted using an averaging model. For this, the mean discharge rate was computed by summing the discharge rates in the $1 \mathrm{~T}$ and $1 \mathrm{~S}$ conditions and dividing by 2. The solid lines in all panels are the lines of unity. Points falling along this line indicate that the actual discharge rate was predicted accurately from the modeled discharge rate.

eye position for the $1 \mathrm{~T}$ and the $2 \mathrm{~T}$ conditions and found that the endpoint accuracy was statistically indistinguishable in these two conditions ( $t$ test, $p=\mathrm{NS}$; data not shown). As a measure of saccade precision, we computed the SD of the endpoints for the $1 \mathrm{~T}$ and $2 \mathrm{~T}$ conditions. We found here also that the SDs were statistically indistinguishable in the two conditions ( $t$ test, $p=$ NS.; data not shown). Finally, we found no significant differences in saccade velocity between the two conditions ( $t$ test, $p=\mathrm{NS}$; data not shown). We drew two conclusions from these results. First, our monkeys were making accurate saccades to the individual targets in the different conditions. Second, variations in saccadic velocity, endpoint accuracy, or precision cannot explain the modulations we observed in the SC neuronal activity.

\section{Timing of SC neuronal activity modulations}

An important, unresolved question is whether the modulations seen in the SC arise from mechanisms within the SC itself or whether they are reflections of processing occurring elsewhere and are simply passed on to the SC. We reasoned that we could explore this by comparing the time course of neuronal activity changes in the different conditions of our task. Moreover, we could compare the timing of neuronal activity changes in visualtonic neurons to the changes in buildup neurons to determine whether the times differed for these two neuronal classes.

To address this, first we compared the time that neuronal activity separated in the initial visual interval of the $1 \mathrm{~T}$ and the $2 \mathrm{~T}$ conditions. Second, we measured the time when the $2 \mathrm{~T}$ and $2 \mathrm{~S}$ activities separated after the cue in the Go/No-Go task. Finally, we compared the statistically significant times (as determined by a permutation test) measured in visual-tonic neurons to those measured in buildup neurons.

We applied ROC analysis to measure time courses. Considering that top-down modulations may influence stimulus interactions in the blocked condition, we used only the data from the interleaved condition that were significantly different in the $1 \mathrm{~T}$ and the $2 \mathrm{~T}$ conditions during the visual interval (Fig. $8 a$, filled blue triangle). We plotted the normalized spike density functions for buildup $(n=16)$ and visual-tonic $(n=5)$ neurons recorded in the 1T and 2T trial types (Fig. 15a). Using the permutation test, we found that the neuronal activity measured in the two conditions separated at a mean time of $132 \mathrm{~ms}$ (Fig. 15a, top, dashed and solid lines) $(p<0.05)$. Visual-tonic neurons had separable curves at a mean time of $146 \mathrm{~ms}$ (Fig. 15a, bottom, dashed and solid lines) $(p<0.05)$. We next compared the time course of separation between the $2 \mathrm{~T}$ and $2 \mathrm{~S}$ trials for the neurons that had a significant difference in the $2 \mathrm{~T}$ and $2 \mathrm{~S}$ conditions (buildup, 25; visual-tonic, 8). We found that buildup neurons had a mean separation time of $127 \mathrm{~ms}$ (Fig. 15b, top, dashed and solid lines) $(p<0.05)$. Visual-tonic neurons reached significance at $152 \mathrm{~ms}$ (Fig. 15b, bottom, dashed and solid lines) $(p<0.05)$.

\section{Discussion}

We recorded from buildup and visual-tonic neurons within the $\mathrm{SC}$ of monkeys during the performance of Go-Go and Go/No-Go saccade tasks. Our experiments differ from previous experiments (Basso and Wurtz, 1998; Paré and Wurtz, 2001) in two important ways. First, the stimuli were presented within single RFs of SC neurons. Second, the cue to make or withhold a saccade to a target was presented at the fixation point rather than in the periphery. This required a shape to location transformation.

We draw two conclusions from our results. First, the influence of saccade selection on SC neuronal activity is similar to the influence of attention on sensory interactions seen in extrastriate cortex neurons. Second, during the sensorimotor transformation of a visual stimulus to a saccade, the activity of SC neurons is dynamic. The readout for saccade generation is initially a vector average and only later becomes winner-take-all. Each of these conclusions will be addressed below.

\section{Stimulus interactions and saccade selection}

Placing two stimuli within a single RF of a V4, V2 (Moran and Desimone, 1985; Motter, 1994a,b; Luck et al., 1997; Reynolds et al., 1999), or middle temporal area MT neuron (Treue and Maunsell, 1996; Recanzone et al., 1997; Seidemann and Newsome, 1999; Recanzone and Wurtz, 2000) reduces the activity of a neuron compared with the presentation of a single stimulus alone. When cued to attend to one of two stimuli, the neuronal activity moves toward the response of the neuron when the attended stimulus is presented in isolation. These results led to the hypothesis that attention acts to change the sensory responsiveness of neurons to stimuli that are not relevant for behavior, the biased competition model of attention (Desimone and Duncan, 1995; Reynolds et al., 1999).

We wanted to determine whether similar stimulus interactions would influence SC neurons and whether a cue to shift gaze would bias the activity of SC neurons in a manner similar to that seen in cortex. Rationale for these ideas comes primarily from three sources. First, an influential hypothesis, called the "motor theory of attention" (Rizzolatti, 1983; Sheliga et al., 1994, 1995; Kustov and Robinson, 1996), posits that commands to shift gaze 


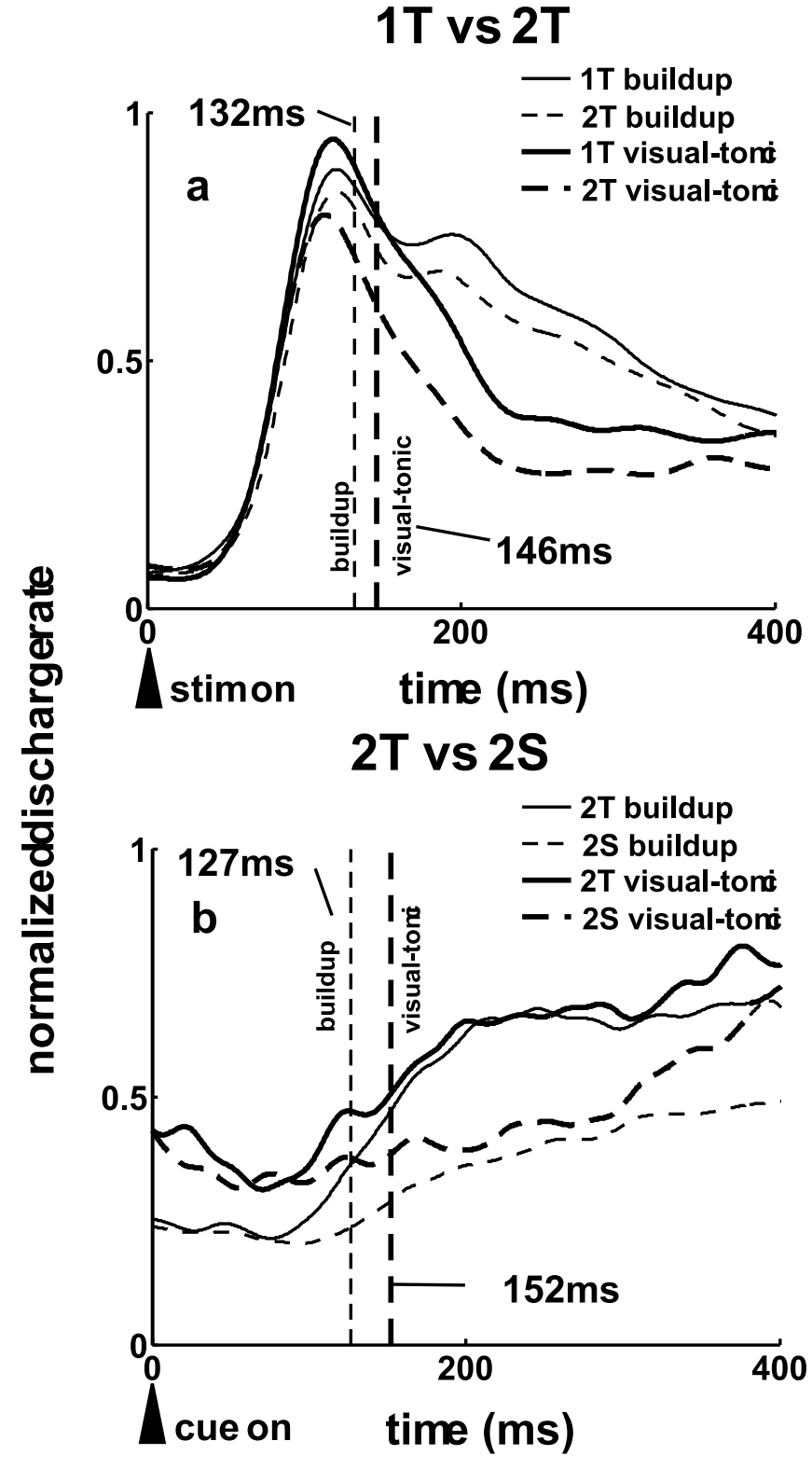

Figure 15. Time course of $\mathrm{SC}$ neuronal discrimination. $\boldsymbol{a}$, Normalized spike density function $(\sigma=15)$ is plotted against time for the $1 \mathrm{~T}$ (solid lines) and 2T (dashed lines) conditions for buildup ( $n=15$ neurons) and visual-tonic neurons $(n=5)$. The activity for each neuron was normalized to its own maximum discharge rate during the visual interval activity. The normalized rates were then averaged across the sample. $\boldsymbol{b}$, The same as in $\boldsymbol{a}$, for the neuronal activity measured after the onset of the cue in the $2 \mathrm{~T}$ (solid lines) and 25 (dashed lines) conditions. The discharge rate of each neuron was normalized to its own maximum rate during the delay 2 interval of the 2T condition. $n=19$ buildup neurons; $n=7$ visual-tonic neurons. Dashed vertical lines in both panels indicate the mean statistically significant time point at which the 2 conditions were distinguishable as determined by ROC and the permutation test (see Materials and Methods).

are also used to shift the location of attention. This theory is based on a large body of psychophysical data (Kowler, 1990; Kowler et al., 1995; Deubel and Schneider, 1996; Schneider and Deubel, 2002). Consistent with psychophysical studies, electrical stimulation of the FEF (Moore and Fallah, 2001, 2004) and SC (Cavanaugh and Wurtz, 2004; Muller et al., 2005) reveals that introducing artificial signals in regions of the brain controlling gaze shifts can enhance sensory processing.

Second, experiments demonstrate the existence of long-range competitive interactions influencing SC neurons (Basso and
Wurtz, 1998; McPeek and Keller, 2002). Electrophysiological studies in cats (Meredith and Ramoa, 1998) and monkeys (Munoz and Istvan, 1998) reveal long-range competitive interactions in SC (but see Özen et al., 2004). Psychophysical experiments suggest that there is competition between saccades and fixation (Walker et al., 1997; Findlay and Walker, 1999), which is thought to be mediated by mutual inhibition between the rostral and caudal SC (Munoz and Wurtz, 1993a,b).

Third, short-range inhibitory mechanisms are likely to exist within the SC (Lee et al., 1997, 2001). Presenting two stimuli in succession, within single RFs of superficial layer SC neurons, results in a suppressed response compared with when one stimulus is presented alone (Rizzolatti et al., 1973, 1974; Wurtz et al., 1980). Anatomical evidence reveals GABA neurons distributed uniformly throughout the SC (Mize, 1992; Behan et al., 2002). The present results demonstrate short-range competitive interactions influencing SC neurons. One hypothesis is that these are mediated by inhibition within the SC. A second hypothesis is that the interactions occur in cortex and are transmitted through the direct connections to the SC (Graham et al., 1979; Fries, 1984; Harting et al., 1992). The time course we measured between the $1 \mathrm{~T}$ and $2 \mathrm{~T}$ conditions is consistent with the hypothesis that inhibitory interactions occur simultaneously in SC and cortex [ 100-150 ms for V4 (Reynolds et al., 1999); 128-140 ms for FEF (Thompson et al., 1996)]. A similar conclusion was drawn for target selection experiments in SC (McPeek and Keller, 2002).

We measured SC neuronal activity after a cue was provided to either make or withhold a saccade, or, to make a saccade to differently shaped targets. Consistent with a biased competition, when a strong stimulus (defined by the preferred location) was cued, neuronal activity increased. When a weak stimulus was cued, the activity also increased, although not as much as when the strong stimulus was cued (Figs. 7, 9, 10, 11). We found an important difference by comparing the sensory interaction index with the selectivity index measured for SC neurons before and after a cue to make a saccade (Fig. 12). When two stimuli were in the RF of SC neurons, the interaction depended on the task demands. If monkeys chose a saccade target that by itself produced a weak response (located on the edge of the RF: probe), the suppression of SC neuronal activity in response to the pair of stimuli was greater after the cue compared with before the cue (Fig. $12 a, b)$. If monkeys chose a saccade target that alone produced a strong response (located at the center of the RF: reference), SC neurons showed less suppression to the pair of stimuli after the cue compared with before the cue (Fig. 12c,d). This is reminiscent of the phenomenon in extrastriate cortex in which sensory interactions are modified by attention (Reynolds and Desimone, 1999, their Figs. 10, 11). We conclude, therefore, that saccade selection, like attention, modifies the influence of stimulus interactions on SC neurons. These results are consistent with the growing body of evidence implicating the persistent activity of SC neurons in attentional processing (Kustov and Robinson, 1996; Cavanaugh and Wurtz, 2004; Ignashchenkova et al., 2004; Muller et al., 2005).

We also found that buildup neurons discriminated faster than visual-tonic neurons. We cannot determine unequivocally the location of visual-tonic neurons but, because of the general finding that they were located more dorsal to the buildup neurons and because of the similarity between these neurons and those recorded by McPeek and Keller (2002), we suspect that these are the neurons originally described by Sparks and Mays (1980), as quasi visual neurons. Our results suggest that visual information reaches buildup neurons before visual-tonic neurons. Because of 
the small number of neurons contributing to the results, additional experiments are required to determine this conclusively.

\section{Implications for population coding in SC}

Models of the SC in saccade generation have been developed primarily using single-point visual stimulation (Lee et al., 1988; Van Opstal and Van Gisbergen, 1989; McIlwain, 1991; Arai et al., 1993a,b, 1994; Van Opstal and Kappen, 1993; Das et al., 1996; Bozis and Moschovakis, 1998; Quaia et al., 1998, 1999). Based on the well known fact that individual saccades are associated with a large population of active SC neurons (Schiller and Koerner, 1971; Wurtz and Goldberg, 1972; Sparks et al., 1976; Sparks and Mays, 1980; Munoz and Wurtz, 1995) and that there are excitatory connections linking large regions of the SC (McIlwain, 1982), the models fall into the general class of distributed coding models (Lee et al., 1988; McIlwain, 1991; Quaia et al., 1998, 1999). Some original models proposed that the activity across the SC map was summed linearly (Ottes et al., 1986). These models failed to replicate the well known behavior of saccade averaging (Findlay, 1982, 1992; Ottes et al., 1984, 1987). When subjects are asked to look toward one of two stimuli, they are frequently inaccurate, as if making a movement to an illusory target located between the two stimuli. To produce more realistic saccade behavior, nearby excitation and remote, lateral inhibitory mechanisms were included in subsequent models (Van Gisbergen et al., 1987; Van Opstal and Van Gisbergen, 1989).

Two issues arise. First, is the divisive inhibition required to produce averaging (Carandini et al., 1997; Britten and Heuer, 1999; Groh, 2001) performed within or downstream of the SC? Second, is the readout of SC activity dynamic? With time or additional information (Coëffé and O'Regan, 1987; He and Kowler, 1989), saccades can be made accurately, rivaling perceptual accuracy (Kowler and Blaser, 1995; Kowler et al., 1995). The results of our experiments provide insight into both of these questions. We found that the response of SC neurons to the presentation of two stimuli was an average of the response of SC neurons to a single stimulus presented alone, consistent with normalization occurring within the SC at least for the visual and the delay-period activity. In light of observations that increasing the size of a visual stimulus does not change neuronal activity in SC (Cynader and Berman, 1972; Goldberg and Wurtz, 1972a), our result also suggests that SC neurons interpret a single large stimulus differently from two individual stimuli.

We also found that the pattern of normalization changed as the trial progressed. Initially, the pattern was characteristic of averaging, but, by the time of the saccade, the activity most closely matched that seen with a single target. This is consistent with the readout of SC activity changing from a vector average to a winner-take-all.

Based on our results, we suggest that saccade averaging may result from the same mechanisms resulting in illusory visual conjunctions (Treisman and Gelade, 1980; Treisman and Sato, 1990; Treisman, 1996), namely, a failure to engage top-down mechanisms regulating bottom-up, stimulus interactions. Recently, it has been suggested that synchronous firing of neuronal populations contributes to the solution of this problem (Singer and Gray, 1995; Reynolds and Desimone, 1999) (but see Shadlen and Movshon, 1999) and that temporal patterns are important for SC function (Brecht et al., 1999, 2004). If so, a top-down selection mechanism may determine which neuronal populations to synchronize or otherwise serve to disambiguate saccade targets when they activate overlapping neuronal populations.

\section{References}

Arai K, Keller EL, Edelman JA (1993a) A spatio-temporal neural network model of saccade generation. In: Proceedings of the IEEE International Joint Conference on Neural Networks, pp 70-74. Amsterdam: Elsevier.

Arai K, Keller EL, Edelman JA (1993b) A neural network model of saccade generation using distributed dynamic feedback to superior colliculus. In: International Joint Conference on Neural Networks (Nagoya), pp 53-56. Amsterdam: Elsevier.

Arai K, Keller EL, Edelman JA (1994) 2-dimensional neural network model of the primate saccadic system. Neural Netw 7:1115-1135.

Basso MA, Wurtz RH (1997) Modulation of neuronal activity by target uncertainty. Nature 389:66-69.

Basso MA, Wurtz RH (1998) Modulation of neuronal activity in superior colliculus by changes in target probability. J Neurosci 18:7519-7534.

Basso MA, Wurtz RH (2002) Neuronal activity in substantia nigra pars reticulata during target selection. J Neurosci 22:1883-1894.

Behan M, Steinhacker K, Jeffrey-Borger S, Meredith M (2002) Chemoarchitecture of GABAergic neurons in the ferret superior colliculus. J Comp Neurol 452:334-359.

Bozis A, Moschovakis AK (1998) Neural network simulations of the primate oculomotor system. III. An one dimensional, one directional model of the superior colliculus. Biol Cybern 79:215-230.

Bradley A, Skottun B, C, Ohzawa I, Sclar G, Freeman R (1987) Visual orientation and spatial frequency discrimination: a comparison of single neurons and behavior. J Neurophysiol 57:755-772.

Brecht M, Singer W, Engel AK (1999) Patterns of synchronization in the superior colliculus of anesthetized cats. J Neurosci 19:3567-3579.

Brecht M, Singer W, Engel AK (2004) Amplitude and direction of saccadic eye movements depend upon the synchronicity of collicular population activity. J Neurophysiol 92:424-432.

Britten KH, Heuer HW (1999) Spatial summation in receptive fields of MT neurons. J Neurosci 19:5074-5084.

Britten KH, Shadlen MN, Newsome WT, Movshon JA (1992) The analysis of visual motion: a comparison of neuronal and psychophysical performance. J Neurosci 12:4745-4765.

Carandini M, Heeger DJ, Movshon JA (1997) Linearity and normalization in simple cells of the macaque primary visual cortex. J Neurosci 17:8621-8644.

Carello CD, Krauzlis RJ (2004) Manipulating intent: evidence for a causal role of the superior colliculus in target selection. Neuron 43:575-583.

Cavanaugh J, Wurtz RH (2004) Subcortical modulation of attention counters change blindness. J Neurosci 24:11236-11243.

Coëffé C, O’Regan JK (1987) Reducing the influence of non-target stimuli on saccade accuracy: predictability and latency effects. Vision Res 27:227-240.

Cohn TE, Green DG, Tanner WJ (1975) Receiver operating characteristic analysis: application to the study of quantum fluctuation effects in optic nerve of Rana pipiens. J Gen Physiol 66:583-616.

Crist CF, Yamasaki DSG, Komatsu H, Wurtz RH (1988) A grid system and a microsyringe for single cell recording. J Neurosci Methods 26:117-122.

Cynader M, Berman N (1972) Receptive-field organization of monkey superior colliculus. J Neurophysiol 35:187-201.

Das S, Keller EL, Arai K (1996) A distributed model of the saccadic system: the effects of internal noise. Neurocomputing 11:245-269.

Desimone R, Duncan J (1995) Neural mechanisms of selective visual attention. Annu Rev Neurosci 18:183-222.

Deubel H, Schneider WX (1996) Saccade target selection and object recognition: evidence for a common attentional mechanism. Vision Res 36:1827-1837.

Dorris MC, Paré M, Munoz DP (1997) Neuronal activity in monkey superior colliculus related to the initiation of saccadic eye movements. J Neurosci 17:8566-8579.

Efron B, Tibshirani RJ (1998) An introduction to the bootstrap, Ed 2. Washington, DC: Chapman and Hall/CRC.

Ferrera VP, Lisberger SG (1995) Attention and target selection for smooth pursuit eye movements. J Neurosci 15:7472-7484.

Ferrera VP, Lisberger SG (1997) Neuronal responses in visual areas MT and MST during smooth pursuit target selection. J Neurophysiol 78:1433-1446.

Findlay JM (1982) Global visual processing for saccadic eye movements. Vision Res 22:1033-1045.

Findlay JM (1992) Programming of stimulus-elicited saccadic eye move- 
ments. In: Eye movements and visual cognition (Rayner K, ed), pp 8-31. Berlin: Springer.

Findlay JM, Walker R (1999) A model of saccade generation based on parallel processing and competitive inhibition. Behav Brain Sci 22:661-721.

Fries W (1984) Cortical projections to the superior colliculus in the macaque monkey: a retrograde study using horseradish peroxidase. J Comp Neurol 230:55-76.

Fuchs AF, Robinson DA (1966) A method for measuring horizontal and vertical eye movement chronically in the monkey. J Appl Physiol 21:1068-1070.

Glimcher PW, Sparks DL (1992) Movement selection in advance of action in the superior colliculus. Nature 355:542-545.

Goldberg ME, Wurtz RH (1971) Changing the behavioral significance of a visual stimulus affects the response of neurons in monkey superior colliculus. Proc Intl Union Physiol Sci 9:209.

Goldberg ME, Wurtz RH (1972a) Activity of superior colliculus in behaving monkey. I. Visual receptive fields of single neurons. J Neurophysiol 35:542-559.

Goldberg ME, Wurtz RH (1972b) Activity of superior colliculus in behaving monkeys. II. Effect of attention on neuronal responses. J Neurophysiol 35:560-574.

Graham J, Lin C-S, Kaas JH (1979) Subcortical projections of six visual cortical areas in the owl monkey, Aotus trivirgatus. J Comp Neurol 187:557-580.

Green DM, Swets JA (1966) Signal detection theory and psychophysics. New York: Wiley.

Groh JJ (2001) Converting neural signals from place codes to rate codes. Biol Cybern 85:159-165.

Harting JK, Updyke BV, Van Lieshout DP (1992) Corticotectal projections in the cat: anterograde transport studies of twenty-five cortical areas. J Comp Neurol 324:379-414.

He P, Kowler E (1989) The role of location probability in the programming of saccades: implications for "center-of-gravity" tendencies. Vision Res 29:1165-1181.

Horwitz GD, Newsome WT (1999) Separate signals for target selection and movement specification in the superior colliculus. Science 284:1158-1161.

Horwitz GD, Newsome WT (2001) Target selection for saccadic eye movements: prelude activity in the superior colliculus during a directiondiscrimination task. J Neurophysiol 86:2543-2558.

Ignashchenkova A, Dicke PW, Haarmeier T, Thier P (2004) Neuronspecific contribution of the superior colliculus to overt and covert shifts of attention. Nat Neurosci 7:56-64.

Judge SJ, Richmond BJ, Chu FC (1980) Implantation of magnetic search coils for measurement of eye position: an improved method. Vision Res 20:535-538.

Keppel G (1991) Design and analysis: a researcher's handbook, Ed 3. Upper Saddle River, NJ: Prentice-Hall.

Kowler E (1990) The role of visual and cognitive processes in the control of eye movement. In: Eye movements and their role in visual and cognitive processes (Kowler E, ed), pp 1-63. Amsterdam: Elsevier.

Kowler E, Blaser E (1995) The accuracy and precision of saccades to small and large targets. Vision Res 35:1741-1754.

Kowler E, Anderson E, Dosher B, Blaser E (1995) The role of attention in the programming of saccades. Vision Res 35:1897-1916.

Krauzlis RJ, Dill N (2002) Neural correlates of target choice for pursuit and saccades in the primate superior colliculus. Neuron 35:355-363.

Kustov AA, Robinson DL (1996) Shared neural control of attentional shifts and eye movements. Nature 384:74-77.

Lee C, Rohrer WH, Sparks DL (1988) Population coding of saccadic eye movements by neurons in the superior colliculus. Nature 332:357-360.

Lee PH, Helms MC, Augustine GJ, Hall WC (1997) Role of intrinsic synaptic circuitry in collicular sensorimotor integration. Proc Natl Acad Sci USA 94:13299-13304.

Lee PH, Schmidt M, Hall WC (2001) Excitatory and inhibitory circuitry in the superficial gray layer of the superior colliculus. J Neurosci 21:8145-8153.

Luck SJ, Chelazzi L, Hillyard SA, Desimone R (1997) Neural mechanisms of spatial selective attention in areas V1, V2, and V4 of macaque visual cortex. J Neurophysiol 77:24-42.

McIlwain JT (1975) Visual receptive fields and their images in superior colliculus of the cat. J Neurophysiol 38:219-230.
McIlwain JT (1982) Lateral spread of neural excitation during microstimulation in intermediate gray layer of cat's superior colliculus. J Neurophysiol 47:167-178.

McIlwain JT (1986) Point images in the visual system: new interest in an old idea. Trends Neurosci 9:354-358.

Mcllwain JT (1991) Distributed spatial coding in the superior colliculus: a review. Vis Neurosci 6:3-13.

McPeek RM, Keller EL (2002) Saccade target selection in the superior colliculus during a visual search task. J Neurophysiol 88:2019-2034.

McPeek RM, Keller EL (2004) Deficits in saccade target selection after inactivation of superior colliculus. Nat Neurosci 7:757-763.

Meredith MA, Ramoa AS (1998) Intrinsic circuitry of the superior colliculus: pharmacophysiological identification of horizontally oriented inhibitory interneurons. J Neurophysiol 79:1597-1602.

Mize RR (1992) The organization of GABAergic neurons in the mammalian superior colliculus. Prog Brain Res 90:219-248.

Moore T, Fallah M (2001) Control of eye movements and spatial attention. Proc Natl Acad Sci USA 98:1273-1276.

Moore T, Fallah M (2004) Microstimulation of the frontal eye field and its effects on covert spatial attention. J Neurophysiol 91:152-162.

Moran J, Desimone R (1985) Selective attention gates visual processing in extrastriate cortex. Science 229:782-784.

Motter BC (1993) Focal attention produces spatially selective processing in visual cortical areas V1, V2, and V4 in the presence of competing stimuli. J Neurophysiol 70:909-919.

Motter BC (1994a) Neural correlates of attentive selection for color or luminance in extrastriate area V4. J Neurosci 14:2178-2189.

Motter BC (1994b) Neural correlates of feature selectivity memory and pop-out in extrastriate area V4. J Neurosci 14:2190-2199.

Muller JR, Philiastides MG, Newsome WT (2005) Inaugural article: microstimulation of the superior colliculus focuses attention without moving the eyes. Proc Natl Acad Sci USA 102:524-529.

Munoz DP, Wurtz RH (1993a) Fixation cells in monkey superior colliculus. I. Characteristics of cell discharge. J Neurophysiol 70:559-575.

Munoz DP, Wurtz RH (1993b) Fixation cells in monkey superior colliculus. II. Reversible activation and deactivation. J Neurophysiol 70:576-589.

Munoz DP, Wurtz RH (1995) Saccade-related activity in monkey superior colliculus. I. Characteristics of burst and buildup cells. J Neurophysiol 73:2313-2333.

Munoz DP, Istvan PJ (1998) Lateral inhibitory interactions in the intermediate layers of the monkey superior colliculus. J Neurophysiol 79:1193-1209.

Ottes FP, Van Gisbergen JAM, Eggermont JJ (1984) Metrics of saccade responses to visual double stimuli: two different modes. Vision Res 24:1169-1179.

Ottes FP, Van Gisbergen JAM, Eggermont JJ (1986) Visuomotor fields of the superior colliculus: a quantitative model. Vision Res 26:857-873.

Ottes FP, Van Gisbergen JAM, Eggermont JJ (1987) Collicular involvement in a saccadic colour discrimination task. Exp Brain Res 66:465-478.

Özen G, Helms MC, Hall WC (2004) The intracollicular neuronal network. In: The superior colliculus: new approaches for studying sensorimotor integration (Hall WC, Moschovakis MA, eds), pp 147-158. Boca Raton, FL: CRC.

Paré M, Wurtz RH (2001) Progression in neuronal processing for saccadic eye movements from parietal cortex area LIP to superior colliculus. J Neurophysiol 85:2545-2562.

Port NL, Wurtz RH (2003) Sequential activity of simultaneously recorded neurons in the superior colliculus during curved saccades. J Neurophysiol 90:1887-1903.

Quaia C, Aizawa H, Optican LM, Wurtz RH (1998) Reversible inactivation of monkey superior colliculus. II. Maps of saccadic deficits. J Neurophysiol 79:2097-2110.

Quaia C, Lefèvre P, Optican LM (1999) Model of the control of saccades by superior colliculus and cerebellum. J Neurophysiol 82:999-1018.

Recanzone GH, Wurtz RH (2000) Effects of attention on MT and MST neuronal activity during pursuit initiation. J Neurophysiol 83:777-790.

Recanzone GH, Wurtz RH, Schwarz U (1997) Responses of MT and MST neurons to one and two moving objects in the receptive field. J Neurophysiol 78:2904-2915.

Reynolds JH, Desimone R (1999) The role of neural mechanisms of attention in solving the binding problem. Neuron 24:19-29. 
Reynolds JH, Chelazzi L, Desimone R (1999) Competitive mechanisms subserve attention in macaque areas V2 and V4. J Neurosci 19:1736-1753.

Reynolds JH, Pasternak T, Desimone R (2000) Attention increases sensitivity of V4 neurons. Neuron 26:703-714.

Rizzolatti G (1983) Mechanisms of selective attention in mammals. In: Advances in vertebrate neuroethology (Ewert J-P, Capranica R, Ingle DJ, eds). New York: Plenum.

Rizzolatti G, Camarda R, Grupp LA, Pisa M (1973) Inhibition of visual responses of single units in the cat superior colliculus by the introduction of a second visual stimulus. Brain Res 61:390-394.

Rizzolatti G, Camarda R, Grupp LA, Pisa M (1974) Inhibitory effect of remote visual stimuli on visual response of cat superior colliculus: spatial and temporal factors. J Neurophysiol 37:1262-1275.

Schall JD (1991) Neuronal activity related to visually guided saccades in the frontal eye fields of rhesus monkeys: comparison with supplementary eye fields. J Neurophysiol 66:559-579.

Schall JD (1995) Neural basis of saccade target selection. Rev Neurosci 6:63-85.

Schall JD, Hanes DP, Thompson KG, King DJ (1995) Saccade target selection in frontal eye field of macaque. I. Visual and premovement activation. J Neurosci 15:6905-6918.

Schiller PH, Koerner F (1971) Discharge characteristics of single units in superior colliculus of the alert rhesus monkey. J Neurophysiol 34:920-936.

Schneider WX, Deubel H (2002) Selection-for-perception and selectionfor-spatial-motor action are coupled by visual attention: a review of recent findings and new evidence from stimulus driven saccade control. Oxford: Oxford UP.

Seidemann E, Newsome WT (1999) Effect of spatial attention on responses of area MT neurons. J Neurophysiol 81:1783-1794.

Shadlen MN, Movshon JA (1999) Synchrony unbound: a critical evaluation of the temporal binding hypothesis. Neuron 24:67-77.

Sheliga BM, Riggio L, Rizzolatti G (1994) Orienting of attention and eye movements. Exp Brain Res 98:507-522.

Sheliga BM, Riggio L, Rizzolatti G (1995) Spatial attention and eye movements. Exp Brain Res 105:261-275.
Singer W, Gray CM (1995) Visual feature integration and the temporal correlation hypothesis. Annu Rev Neurosci 18:555-586.

Sparks DL, Mays LE (1980) Movement fields of saccade-related burst neurons in the monkey superior colliculus. Brain Res 190:39-50.

Sparks DL, Holland R, Guthrie BL (1976) Size and distribution of movement fields in the monkey superior colliculus. Brain Res 113:21-34.

Thompson KG, Hanes DP, Bichot NP, Schall JD (1996) Perceptual and motor processing stages identified in the activity of macaque frontal eye field neurons during visual search. J Neurophysiol 76:4040-4054.

Treisman A (1996) The binding problem. Curr Opin Neurobiol 6:171-178.

Treisman A, Gelade G (1980) A feature-integration theory of attention. Cognit Psychol 12:97-136.

Treisman A, Sato S (1990) Conjunction search revisited. J Exp Psychol Hum Percept Perform 16:459-478.

Treue S, Maunsell JH (1996) Attentional modulation of visual motion processing in cortical areas MT and MST. Nature 382:539-541.

Van Gisbergen JAM, Van Opstal AJ, Tax AAM (1987) Collicular ensemble coding of saccades based on vector summation. Neuroscience 21:541-555.

Van Opstal AJ, Kappen H (1993) A two-dimensional ensemble coding model for spatial-temporal transformation of saccades in monkey superior colliculus. Network 4:19-38.

Van Opstal AJ, Van Gisbergen JAM (1989) A nonlinear model for collicular spatial interactions underlying the metrical properties of electrically elicited saccades. Biol Cybern 60:171-183.

Walker R, Deubel H, Schneider WX, Findlay JM (1997) Effect of remote distractors on saccade programming: evidence for an extended fixation zone. J Neurophysiol 78:1108-1119.

Wurtz RH, Goldberg ME (1972) Activity of superior colliculus in behaving monkey: III. Cells discharging before eye movements. J Neurophysiol 35:575-586.

Wurtz RH, Richmond BJ, Judge SJ (1980) Vision during saccadic eye movements. III. Visual interactions in monkey superior colliculus. J Neurophysiol 43:1168-1181. 